\title{
Towards optimizing the deployment of optical access networks
}

\author{
Martin Grötschel · Christian Raack · Axel Werner
}

Received: 2 April 2013 / Accepted: 24 September 2013 / Published online: 5 December 2013 (C) Springer-Verlag Berlin Heidelberg and EURO - The Association of European Operational Research Societies 2013

\begin{abstract}
In this paper we study the cost-optimal deployment of optical access networks considering variants of the problem such as fiber to the home (FTTH), fiber to the building (FTTB), fiber to the curb (FTTC), or fiber to the neighborhood (FTTN). We identify the combinatorial structures of the most important sub-problems arising in this area and model these, e.g., as capacitated facility location, concentrator location, or Steiner tree problems. We discuss modeling alternatives as well. We finally construct a unified integer programming model that combines all sub-models and provides a global view of all these FTTx problems. We also summarize computational studies of various special cases.
\end{abstract}

Keywords FTTx $\cdot$ FTTH $\cdot$ FTTB $\cdot$ FTTC $\cdot$ FTTN $\cdot$ Telecommunications $\cdot$ Access networks $\cdot$ Passive optical networks $\cdot$ Network design $\cdot$ Routing $\cdot$ Energy efficiency

Mathematics Subject Classification (2000) $\quad 90 \mathrm{~B} 18 \cdot 90 \mathrm{~B} 80 \cdot 90 \mathrm{C} 11 \cdot 90 \mathrm{C} 35$

\section{Introduction}

Today optical transmission is the standard technology in backbone and metro region telecommunication networks. However, in local access networks (LAN), which connect customers or business units with the next metropolitan aggregation point, optical

M. Grötschel · A. Werner $(\bowtie)$

Zuse Institute Berlin (ZIB), Takustraße 7, 14195 Berlin, Germany

e-mail: werner@zib.de

M. Grötschel

e-mail: groetschel@zib.de

C. Raack

Atesio GmbH, Bundesallee 89, 12161 Berlin, Germany

e-mail: raack@atesio.de 
transmission is still not ubiquitous. In many countries the data flows in this so-called "last-mile" of the network hierarchy from the backbone towards the end-user are still electrically switched. They are transported over copper cables from old telephone networks via digital subscriber line (DSL) technology. Bit rates beyond $50 \mathrm{Mbps}$ can, in principle, also be obtained using the TV cable infrastructure (co-axial cables) or fourth generation wireless technology (long-term evolution-LTE). Optical fiber, though, is superior to copper cables and radio access in many aspects: (a) the high data rates and the available bandwidth ensure a future-proof scalability of optical transmission, which is very important in light of the increasing demand for new multimedia services; (b) the lower energy consumption benefits efforts to increase sustainability in telecommunications; (c) the immunity to electromagnetic interference and crosstalk and the practically non-existent radiation increase security and efficiency of the networks and allow for much longer reach of signal transmission. These advantages drive network operators to renew their infrastructure in the last mile. The associated efforts involve extremely large investments and heavy construction work; hence, it is necessary to employ suitable optimization models to plan future broadband optical access networks.

Balakrishnan et al. (1991, p. 248) stated in their paper on the capacity expansion in access networks in 1991, at a time where fiber-optic technology was already used for long-distance transmissions, but in a mere experimental stage for access networks:

"[W]e do not represent the unique characteristics of fiber optic transmission in great detail, particularly since this technology is still evolving. When the technology develops further and telephone companies gain experience with deploying it, network planners might require more sophisticated models to distinguish fiber optic transmission from conventional electrical transmission".

Now, more than 20 years later, knowledge in this area has expanded significantly. The deployment of optical fiber access networks has started. This results in an increasing demand for tools that provide feasible network solutions and for methodology that can be used to plan and optimize the deployment. We, thus, believe that it is valuable to look into and survey "more sophisticated models" that have been proposed.

\section{Practical background}

We first gather some details of the technical and planning background that are important to understand the nature of the modeling approaches described in this paper, and explain the unavoidable abbreviations used in the field. For more information on practical issues we refer to technical documents, such as Rigby (2011) or Keiser (2006).

We consider the planning of optical fiber access networks, which means that we want to connect potential clients (business customers, buildings, houses, neighborhoods) in a local area to an aggregation point, commonly called central office (CO) or point of presence (POP), by optical fiber, see Fig. 1. Local area, in this context, refers to a small city or a district in a larger city spanning at most a few kilometers in diameter. A single $\mathrm{CO}$ might serve several thousand clients in densely populated regions. We concentrate exclusively on the design of the client access network, that is, we ignore 


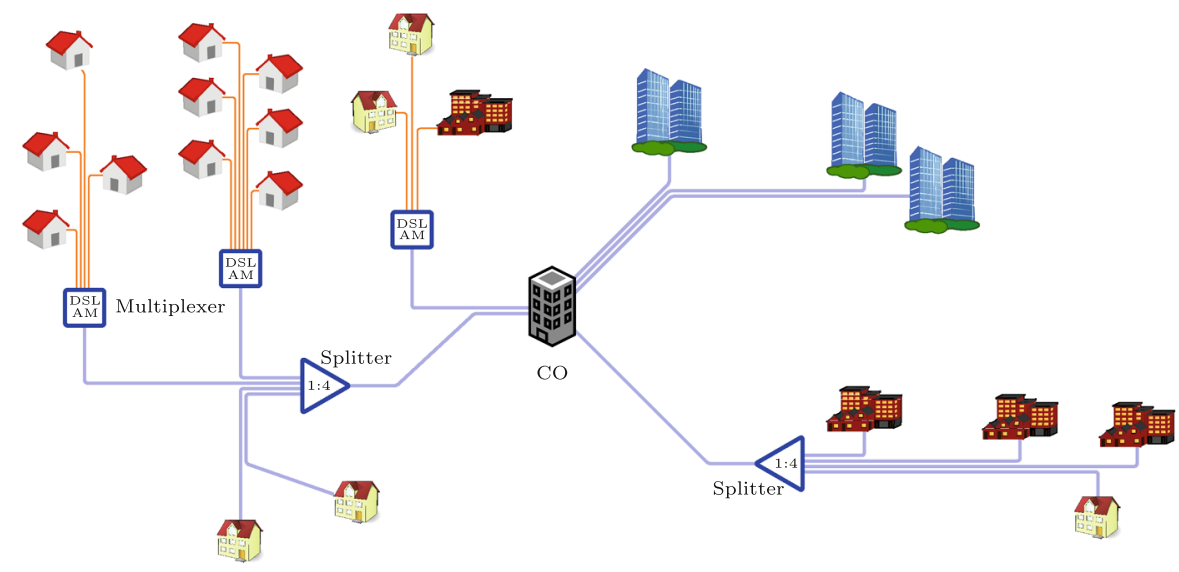

Fig. 1 Deployment of an FTTx network: some customers are connected to the CO via FTTH/FTTB and point-to-multipoint (lower left and lower right). There are direct point-to-point FTTB connections (upper right) and various FTTC connections using existing copper cables (upper left). Optical fiber connections are shown in blue, copper cable in red (color figure online)

the network between the aggregation points of different metropolitan regions as well as the long-distance, high-capacity backbone of the telecommunication network.

Planning the optical access is colloquially referred to as Fiber-To-The-x or simply FTTx, where the placeholder "x" stands for the possible client-side termination points of the fiber connection: usually the home of a customer (Fiber-To-The-Home or FTTH), the (basement of a) building where customers reside (Fiber-To-The-Building or FTTB), or a street cabinet somewhere at the curb (Fiber-To-The-Curb or FTTC) or in the neighborhood (Fiber-To-The-Neighborhood or FTTN). Figure 1 shows possible fiber termination points.

A central issue in this context is that the signal transmission between the $\mathrm{CO}$ and the fiber termination point is passive, that is, end-to-end fiber connectivity without intermediate regeneration of optical signals has to be provided. This implies that technology-dependent maximal lengths of fiber connections have to be respected. Another important difference to the capacity expansion of existing copper (telephone) networks is that, except for the potential use of existing duct systems, one typically has to face green-field planning. In particular, we may not assume that the underlying network already has a tree structure.

Whatever it takes to connect (all or part of) the customers in a given deployment area to one or more central offices using optical fiber (and potentially existing copper infrastructure) is the topic of FTTx network planning; see Fig. 2 for illustration. This involves digging trenches, laying fibers, cables, and ducts, and providing sufficiently capacitated node equipment.

Besides the question what the " $\mathrm{x}$ " should be, there are various other strategies how to implement an FTTx network. One fundamental distinction is between pointto-point (P2P) networks, where each customer is supplied with its own individual fiber, and point-to-multipoint (P2MP) networks, where signals to different customers are transmitted on the same fiber starting at the $\mathrm{CO}$ and split up at some point in 

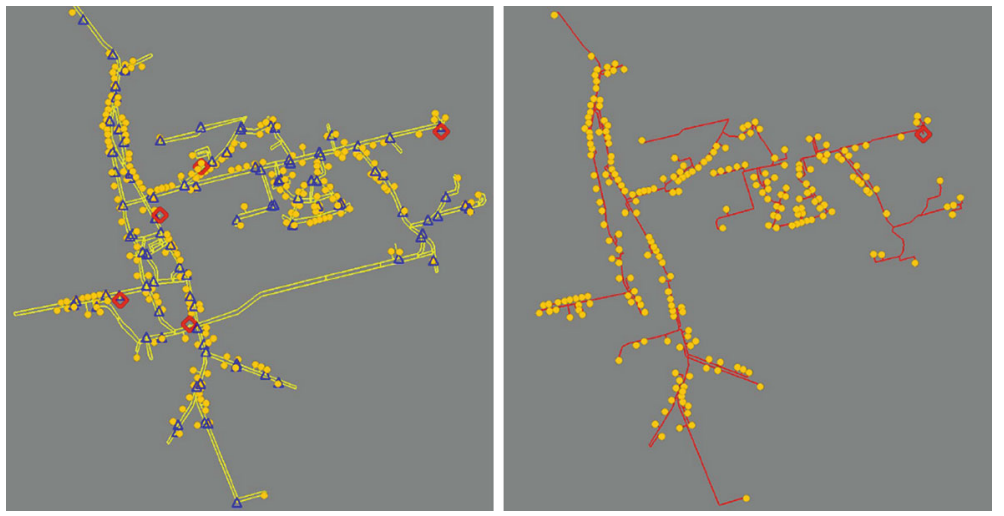

Fig. 2 Example of an input network (left) and a solution (right) for a certain geographical area: diamond icons symbolize (potential) CO locations, equipment (splitters or cabinets) can be installed at triangle nodes; in the solution no triangle nodes have been chosen, so this represents a P2P FTTH network (also cf. Fig. $6)$.

the network, see Fig. 1. P2MP networks are commonly called passive optical networks (PON). This term is somewhat misleading in our context, since also P2P FTTH networks are "passive optical", which is why we avoid to use the term PON in this paper.

On the "CO-end" of the optical fiber access resides an optical line terminal (OLT), a port generating the optical signals for the connected customers and emitting them into the fiber. Further installations are usually made to hold the active (i.e. powerconsuming) devices at the $\mathrm{CO}$ and assign fiber connections to ports. The "customerend", where optical signals are converted into electrical signals is usually defined by a so-called optical network unit (ONU). In an FTTC network the signals are then further distributed by a DSL access multiplexer (DSLAM), which generates individual electrical (DSL) signals that travel the remaining distance to the customer over copper line, see Fig. 1.

While in a point-to-point scenario a network planner "only" has to decide about the optical connections between COs and customers, in a point-to-multipoint architecture further devices - and therefore placement decisions - have to be incorporated. Current practice in optical access networks uses time-division multiplexing (TDM), where the individual customer-bound signals are multiplexed in the time domain. The OLT at the $\mathrm{CO}$ is responsible for assigning bandwidth and time-slots to the customer-side ONUs. The division of the optical signal into multiple copies is done by optical splitters. A splitter consists of elements that passively (i.e. on the optical level, without the need for electric power) split an optical signal into two identical copies (with losses in signal strength, though). By cascading such elements, 1: $\theta$ splitters are realized, where in practice splitting ratios $\theta \in\{2,4,8,16,32,64,128\}$ are used. Eventually, the ONU at each of the customers connected to one splitter sees the same signal and extracts the transmitted information from its assigned time slot through embedded address labels.

A more powerful method of sharing fibers is wavelength-division multiplexing (WDM), where the signals for various customers are encoded into different frequencies in a multicolored optical signal; then the role of splitters is taken by wavelength 

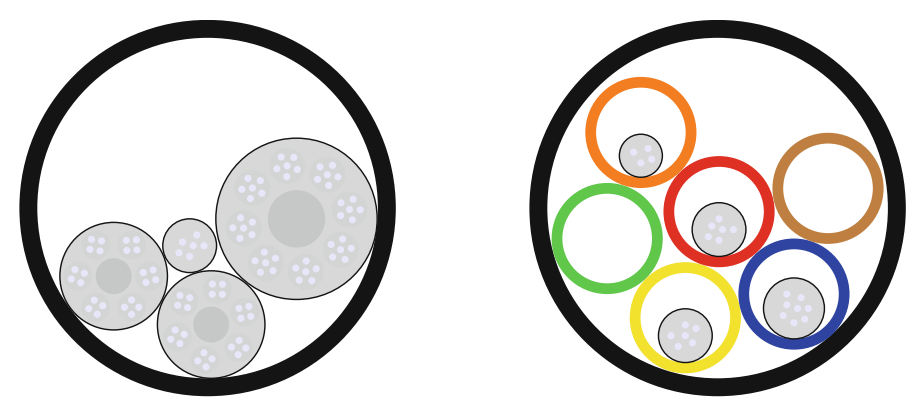

Fig. 3 Cross-section of cable and duct installations (also see Rigby 2011). Left four cables of various sizes $(6,24$, and 48 fibers) in a duct-bigger cables contain a core and have fibers organized in bundles. Right six micro-ducts in a duct, of which four are filled with micro-cables - two more micro-cables can be blown into the empty micro-ducts

filters, so-called optical add-drop multiplexers. While nowadays WDM is successfully implemented in the backbone of nation-wide networks, this technology is still too costly to be implemented on a large scale in local access networks. However, because of the straight-forward scalability it is seen as the method of choice to upgrade existing optical fiber access networks in the future.

Integrating splitters into the network, but also concatenating fibers is a laborious process, since it has to be implemented without too much damage to the integrity of the fiber and its capability to transmit signals with minimal loss. This can be done with sophisticated splicing techniques (see Rigby 2011). It is further complicated by the fact that fibers cannot be laid out individually. They always come in bundles, organized in cables and ducts, which in turn may be embedded in larger duct systems, see Fig. 3. Terminating a major cable and splitting it up into several smaller ones requires splicing each single fiber and subsequently storing the fibers in a safe and organized way in so-called joint closures. To avoid splicing to some degree, there is the possibility to use micro-duct and -cable systems instead of conventional cables (cf. Fig. 3). Here, easy-to-connect small ducts (or bundles of ducts) are laid out, able to accommodate a single cable. The big advantage is that micro-ducts can be deployed, initially left empty, and then filled with a micro-cable at a later point in time, without the need for trenching anew and splicing in between-known as blowing in the micro-cable.

Further considerations have to be taken into account when planning the deployment of real-world networks.

- For technical reasons and because of the passive nature of optical access networks, fiber lengths between central offices and customers have to be limited. Similarly, the length over which a micro-cable can be blown into a micro-duct may not exceed a certain threshold.

- A network expansion plan might be requested that extends over multiple time-periods.

- A difficult issue is whether a planned network is not only optimal with respect to investment costs (CAPEX), but also results in justifiably low operational costs (OPEX).

- Finally, there is always the question, how to obtain and preprocess the input data. 
It is virtually impossible to integrate all practical side-constraints and develop an all-encompassing model for FTTx network planning. But this is also not asked for in practice. Instead, planners typically wish to study several use-cases and scenarios emphasizing different aspects. It depends on the deployment area, on the available technologies, and last but not least on the network operator which planning goals should be focused on and which objective should be optimized. In the following we will work out mathematical aspects that we believe represent the main challenges and combinatorial structures a planner has to face. The models presented here are most likely those that should be considered, together with relaxation and decomposition techniques, and possibly extended in multi-objective settings, when designing algorithmic frameworks and planning tools.

\section{Mathematical problems involved}

There are numerous discrete decisions to be made in the deployment of optical access networks. The exchange of the whole cable infrastructure involves heavy construction activities with large capital expenditures from digging trenches and laying thousands of cable kilometers. On the other hand, network survivability in case of fiber breakages is not a critical side-constraint in access networks, since only a relatively small number of customers gets potentially disconnected, compared to cable damages in the backbone. This combination of high construction costs and low survivability requirements yields that, for the layout of an access network, tree structures are the topology of choice. Thus, one of the most critical optimization (sub)problems is to create a

Steiner Tree: Decide about a tree network (by digging or using existing trenches and ducts) for laying fibers and connecting customers and COs.

An ideal access network provides every customer with a dedicated optical signal, which results in an FTTH deployment implementing point-to-point connections. The assignment of customers to COs and the installation of sufficient equipment at COs creates a second combinatorial sub-problem also inherent to the deployment of access networks:

Capacitated facility location: Decide about the location of COs and equip these with sufficient capacity to meet the fiber demand of the customers. Decide which customer to connect to which $\mathrm{CO}$ by fiber.

There are mainly two approaches to decrease the investment for the cost-intense pointto-point FTTH deployment. First, the existing copper cable infrastructure may be partially reused by moving the ONU, the fiber termination point, away from the customer to the base of the building (FTTB) or to a DSL access multiplexer in a street cabinet (FTTC). Second, fibers may be shared by several customers using a P2MP architecture, which effectively results in installing concentrators (splitters and/or wavelength filters). In general, there might be several levels of concentration. Both, moving the fiber termination point and splitting the signals introduce a trade-off between the capital and operational cost for the network operator and the quality of service for the customer, more precisely, the bit rate avail- 
able via the customer's connection. Regarding the structure of the optimization problems, for FTTH or FTTB the fiber termination point mainly decides about the demand of the corresponding customer node: In case of FTTH, individual households act as customers, for FTTB the building becomes the customer with an aggregated fiber demand. For FTTC/FTTN, however, the installation of cabinets and the assignment of customers to cabinets may be a substantial part of the optimization problem, which leads to a generalization of the mentioned facility location subproblem:

Two-level capacitated facility location: Decide about the location of COs and cabinets with sufficient capacity. Assign customers to cabinets and cabinets to COs. The (bit rate) demand of the individual customers should be met.

With respect to optimization models we speak of FTTH instead of FTTH/FTTB and of FTTC instead of FTTC/FTTN in the following.

Combining the aspects of (two-level) facility location and optimizing the underlying Steiner tree leads to so-called (two-level) connected (capacitated) facility location problems. Another level of complexity is introduced by concentrators in point-tomultipoint networks, which add the notion of signal concentration and splitting ratios to the optimization problem:

Multi-level concentrator location: Decide about the location of COs with sufficient capacity and the location of concentrators with given splitting ratios. Connect customers to COs over potentially several levels of concentration. The (bit rate) demand of the individual customers should be met.

Note that "concentrator location" is very often used as a synonym for facility location in the literature, which is a single-level problem in its pure form. We will instead use the term concentrator location only if there are at least two levels of signal flows (one level of actual concentration/splitting) in the model.

The mentioned tasks can get remarkably complicated if the various capacity restrictions are to be respected. In fact, the actual installation of capacity is typically ignored or simplified in the high-level planning phase but of utmost importance to the practical deployment. In general, we may distinguish capacity at network nodes (customer, concentrator, COs), comprising the design of practical devices such as OLTs, ONUs, splitters, joint closures, or street cabinets, and capacity on links or paths in the network, that is, the organization of the optical fiber, cable, and duct systems.

Capacity coverage, node equipment installation: Given (fiber and bit rate) capacity requirements at network nodes, provide a feasible equipment installation plan. Capacity coverage, path equipment installation: Given routes for connecting customers, concentrators, and COs, compute a feasible cable and duct installation plan that provides the required capacity and ensures the end-to-end connectivity by fiber.

Among all network solutions that meet the mentioned requirements, we usually look for a cost-optimal solution in terms of capital expenditure. Additionally, the objective might include terms accounting for operational costs or expected revenue. The 
aim might even be to minimize several, possibly conflicting, objectives simultaneously, which then leads to multi-objective optimization models. The single-objective problems arising are already very difficult, and therefore, we will not touch upon multi-objective issues such as finding "efficient solutions" or the set of "Pareto optimal points".

\section{Outline of this work}

In the following we study the overall problem of planning the cost-optimal deployment of optical access networks, considering the above mentioned aspects. In "Modeling FTTx problems", we discuss modeling alternatives and introduce the general problem as a combination of capacitated facility location, concentrator location, and Steiner tree problems, incorporating node and link equipment installation. Further aspects that might be important, such as length restrictions, operational costs, etc. are mentioned at the end of the section.

We formulate the models in a new and comprehensive way. Of course, inspiration is taken from the relevant and recent literature that focuses on various special aspects of the FTTx network planning problem. This literature is discussed in "Literature survey". Several of the publications contain computational results, which are gathered in an overview. The paper closes with a summary.

\section{Modeling FTTx problems}

In the following, we iteratively build up a model for the FTTx network planning problem, attempting to integrate as many practically relevant issues as possible. Applying the more extended versions of this model in practice to realistic problem sizes will most certainly lead to computationally unsolvable integer programs - at least for the current "solution machinery". However, we mention possible modifications that yield better applicable formulations and which, in several cases, have in fact been applied in the literature, see "Literature survey".

We assume that we are initially given an undirected simple graph $H=\left(V^{\prime}, E\right)$ representing the deployment area as an abstract network. Along the edges of $H$ connections can be realized; edges will mostly originate from street sections which have to be trenched when used, but might also represent possible crossings of rivers, rail lines, aerial connections, existing duct lines or other usable infrastructure like gas pipes, subway tunnels, or sewers. The nodes of $H$ are those locations where the necessary devices can be installed; in principle, the equipment installable at a given node may be restricted, but we do not assume this in general. The overall task is to select from $H$ certain subgraphs specifying where connections to customers should be laid out, where and how many of which components should be installed, and possibly also when during the deployment period this should happen. The objective is to minimize total investment costs, possibly accounting for expected revenues and/or operational costs. Extensions to multi-criteria models can be obtained by adding further objectives, which we do not state explicitly. 


\section{Connectivity and $\mathrm{CO}$ opening}

Since deploying fiber access networks usually results in tree topologies and typically entails substantial cost in digging up streets and laying ducts, the classical Steiner tree problem (Chopra and Rao 1994a,b; Garey and Johnson 1979) lies at the very core of the problem. From the given undirected graph $H$ we derive a directed simple graph $G=(V, A)$ by replacing each edge $e \in E$ with two anti-parallel arcs (having the end-nodes of $e$ ) and adding an artificial root node $r$ with a directed connection to all potential CO locations $V_{0} \subseteq V^{\prime}$. More precisely, we define $V:=V^{\prime} \cup\{r\}$ and $A:=A_{0} \cup\{(i, j),(j, i): e=i j \in E\}$ with $A_{0}:=\left\{(r, i): i \in V_{0}\right\}$. If we speak of paths in $G$ in this paper, we refer to simple directed paths. We use directed graphs here for notational simplicity but also knowing that the resulting linear programming formulations for models involving trees are stronger than their undirected counterparts in a computational sense (that can be made precise but we will not discuss this here), see (Chopra and Rao 1994a; Gollowitzer and Ljubić 2011). We formulate the basic model in terms of a single commodity flow; there are numerous other (and in some cases better) formulations, but for the subsequent extension of the model this flow formulation seems to be a suitable choice.

We consider the following variables:

- integer flow variables $f_{a}^{1}$ for the number of fibers routed on $\operatorname{arc} a \in A$,

- $0 / 1$ trenching variables $z_{a}$ for opening a trench on arc $a \in A \backslash A_{0}$,

- $0 / 1 \mathrm{CO}$ variables $z_{a}$ for opening a $\mathrm{CO}$ at the target node of $a \in A_{0}$.

Let further $d_{i}^{1} \in \mathbb{Z}_{+}$be the fiber demand at customer node $i \in V \backslash\{r\}$. We use the upperindex notation $f_{a}^{1}$ and $d_{i}^{1}$ instead of $f_{a}$ and $d_{i}$ because these represent first-level fibers, in contrast to the second-level fibers introduced below. For $e=i j \in E$ let $\kappa_{e}>0$ be the cost for trenching (or using existing infrastructure) along the edge between nodes $i$ and $j$. We set $\kappa_{a}:=\kappa_{i j}$ if either $a=(i, j)$ or $a=(j, i)$. If $a=(r, i) \in A_{0}$ then $\kappa_{a}$ refers to the cost of opening a $\mathrm{CO}$ at node $i$. Similarly, assume a cost of $\bar{\kappa}_{a}>0$ for providing a single fiber on arc $a \in A$. This cost usually depends on the length of $a$ if $a \in A \backslash A_{0}$ and might account for the fiber port cost at the CO if $a \in A_{0}$. We restrict the maximal number of fibers routed on arc $a \in A$ by $c_{a} \geq 0, c_{a}$ integral. A basic point-to-point model minimizing the cost for realizing a tree of trenches, opening COs, and laying out fibers such that all customers are connected to a $\mathrm{CO}$ then reads as

$$
\begin{array}{cc}
\min \sum_{a \in A} \kappa_{a} z_{a}+\sum_{a \in A} \bar{\kappa}_{a} f_{a}^{1} & \\
\text { s.t. } \sum_{a \in \delta^{-}(i)} f_{a}^{1}-\sum_{a \in \delta^{+}(i)} f_{a}^{1}=d_{i}^{1} & \forall i \in V \backslash\{r\}, \\
f_{a}^{1} \leq c_{a} z_{a} & \forall a \in A, \\
f_{a}^{1} \in \mathbb{Z}_{+} & \forall a \in A, \\
\sum_{a \in \delta^{-}(i)} z_{a} \leq 1 & \forall i \in V \backslash\{r\},
\end{array}
$$




$$
z_{a} \in\{0,1\} \quad \forall a \in A .
$$

This is similar to classical directed Steiner tree formulations (Chopra and Rao 1994a) with additional flow cost and so-called single-commodity fixed-charge network design models, see for instance (Ortega and Wolsey 2003). The flow equations (1) guarantee that the demand is satisfied by a fiber flow from the root to the customers. Constraints (2) ensure that trenches are opened to lay out the requested fibers. By the degree constraints (4), any optimal solution to this model corresponds to an arborescence in $G$ directed away from the root $r$, which in turn (after removing the root node) corresponds to a Steiner forest in the original network. In fact, this is true even if we remove (4), as long as $c_{a}$ is large, more precisely, as long as $c_{a} \geq \sum_{i \in V} d_{i}^{1}$ for all $a \in A$. It is reasonable to assume this for all $a \in A \backslash A_{0}$ that correspond to trenchable network sections, since usually a single trench can accommodate a huge amount of fiber. However, for $a \in A_{0}$, the value $c_{a}$ refers to the fiber capacity of the corresponding $\mathrm{CO}$, which cannot be guaranteed to cover the fiber demand of all customers in the area. The degree constraints (4) force a tree solution and hence ensure that the fibers of the same customer are assigned to the same CO. Constraints (4) have, for instance, been used by Koch and Martin (1998) to solver Steiner tree problems (cf. also Bley et al. 2013). They may clearly be strengthened to equality constraints for nodes with positive demand.

Notice that in the model above, as well as in the following, we require integrality of the fiber flow variables. These integrality constraints can be relaxed since we force a tree by Constraints (4) and deal with integer demand values. This already ensures the integrality of the flow in all feasible solutions to (1), (2), (3), (4) and (5) and also in all optimal solutions of the models presented below. The situation is slightly more involved if (4) is skipped, but under certain assumptions the integrality restriction can still be relaxed.

\section{Signal splitting}

Using the Steiner arborescence model (1), (2), (3), (4) and (5) above, every customer $i \in V$ with $d_{i}^{1}>0$ is connected directly to the CO by fiber, that is, we have modeled a point-to-point scenario. In contrast, in a point-to-multipoint scenario, by introducing splitters and signal splitting, different customers may share the same fiber from the $\mathrm{CO}$, which saves fiber kilometers, see Fig. 1.

In the following we assume that a splitter is a device that is able to split the signal on a single fiber coming from the $\mathrm{CO}$ at a fixed splitting ratio $1: \theta$ with $\theta \in \mathbb{Z}, \theta>0$. That is, a splitter is connected to the CO by one first-level fiber and to at most $\theta$ customers by second-level fiber. Splitters can be installed in multiples at every node $i \in V \backslash\{r\}$ at a cost of $\kappa_{i}>0$ per splitter.

Clearly, there is a trade-off between a point-to-point scenario and a point-tomultipoint scenario with respect to the cost for fiber and the cost for splitters. In principle, one might let the resulting revenue decide which type of connection is used for a given customer. We will consider such revenue models below. Let us, however, start with a simpler setting where every node $i \in V \backslash\{r\}$ has a targeted demand for first-level fibers of $d_{i}^{1} \in \mathbb{Z}_{+}$(without signal splitting) and a targeted demand for 
second-level (split) fibers of $d_{i}^{2} \in \mathbb{Z}_{+}$. The requested first-level and second-level fibers do not differ physically, they only differ in the available data rate for the customer. We introduce the following additional variables:

- integer flow variables $f_{a}^{2}$ for the number of second-level fibers routed on arc $a \in A$, with $f_{a}^{2}:=0$ for $a \in A_{0}$,

- integer splitter variables $y_{i}$ for the number of splitters installed at nodes $i \in V$, with $y_{r}:=0$ for the artificial root node $r$.

Replacing the fiber flow in the formulation (1), (2), (3), (4) and (5) by a two-level fiber flow yields a basic FTTH P2MP model:

$$
\begin{array}{cl}
\min \sum_{a \in A} \kappa_{a} z_{a}+\sum_{i \in V} \kappa_{i} y_{i}+\sum_{a \in A} \bar{\kappa}_{a}\left(f_{a}^{1}+f_{a}^{2}\right) & \\
\text { s.t. } \sum_{a \in \delta^{-}(i)} f_{a}^{1}-\sum_{a \in \delta^{+}(i)} f_{a}^{1}=d_{i}^{1}+y_{i} & \forall i \in V \backslash\{r\}, \\
\sum_{a \in \delta^{-}(i)} f_{a}^{2}-\sum_{a \in \delta^{+}(i)} f_{a}^{2} \geq d_{i}^{2}-\theta y_{i} & \forall i \in V, \\
f_{a}^{1}+f_{a}^{2} \leq c_{a} z_{a} & \forall a \in A, \\
f_{a}^{l} \in \mathbb{Z}_{+} & \forall a \in A, l=1,2, \\
\sum_{a \in \delta^{-}(i)} \leq 1 & \forall i \in V \backslash\{r\}, \\
z_{a} \in\{0,1\} & \forall a \in A, \\
y_{i} \in \mathbb{Z}_{+} & \forall i \in V .
\end{array}
$$

In this model, the generalized flow constraints (7) ensure that second level fiber demand at the customer is satisfied by installing splitters and routing the fibers from the splitter to the customer. To see this, consider the slack $s_{i} \geq 0$ of constraint (7) for $i \in V$ with

$$
s_{i}:=\sum_{a \in \delta^{-}(i)} f_{a}^{2}-\sum_{a \in \delta^{+}(i)} f_{a}^{2}-d_{i}^{2}+\theta y_{i} \geq 0
$$

and note that by flow conservation we have

$$
\sum_{i \in V}\left(s_{i}+d_{i}^{2}-\theta y_{i}\right)=0
$$

That is, in case of positive second-level fiber demand, there must exist sufficiently many opened splitters with sufficiently many ports. Clearly the value $s_{i}$ corresponds to the unused fiber ports at the splitters provided at node $i \in V$. Constraints (6) guarantee that first-level fiber demand is satisfied directly from one of the $\mathrm{CO}$ locations to the customer (without splitting) and also that every splitter is fed by a single fiber coming from a CO, see Fig. 1. Recall that first-level fiber is supplied by the artificial root node $r$ in our model. If $d_{i}^{2}=0$ for all $i \in V$, then $f_{a}^{2}$ and $y_{i}$ can be set to 0 and 
we obtain the original single-level Steiner arborescence formulation. If, on the other hand, $d_{i}^{1}=0$ for all $i \in V$, then every signal from the CO is split on the way to the customer. Notice that second-level fibers of the same customer might in principle be assigned to different splitters at potentially different nodes.

The above model appears naturally in the context of so-called Concentrator Location problems. The notion of multiple levels of flow with hierarchies of signal flow concentration and flow conservation constraint systems similar to (6) and (7) has been considered already by Balakrishnan et al. (1991) for the capacity expansion in analog and digital telephone access networks. The same approach for fiber flows in P2MP FTTH networks has recently been taken by Bley et al. (2013), who provide a Lagrange decomposition into first-level and second-level part, and by Chardy et al. (2012) and Hervet and Chardy (2012) for several levels of passive optical splitters.

It is straightforward to extend this model to more than one level of splitters as in Balakrishnan et al. (1991), Chardy et al. (2012) and Hervet and Chardy (2012). However, in practice a more frequently implemented scenario for multiple levels of splitting is to place splitters directly at the COs or/and at the customer locations (e.g. in areas with big apartment buildings); this can be modeled simply by preprocessing the demand values. Similarly, the use of different splitter sizes (on one or more levels) could, in principle, also be formulated, cf. Eira et al. (2012), Carpenter et al. (2001) and Bley et al. (2013).

\section{Backfeed}

Since we use a directed formulation here and because we include the tree forcing constraints (10), there is a unique directed fiber path from the $\mathrm{CO}$ to every customer in every feasible solution to (6), (7), (8), (9), (10), (11) and (12). First and second-level fiber always 'flow' into the same direction. This in particular means that our model does not allow for backfeed (cf. Balakrishnan et al. 1991), i.e., connecting a customer to a splitter that does not lie in the direction of the CO, see Fig. 4. In this case, first and second-level fiber would flow into opposite directions on some edge $e \in E$ of the original network $H=\left(V^{\prime}, E\right)$.

Forbidding backfeed might be an undesired restriction for practical scenarios, in particular if splitters are costly and cannot be installed at all locations. In principle, backfeed could be allowed by using an undirected Steiner tree formulation. Also relaxing Constraint (10) would lead to a more flexible assignment of customers to splitters with solutions allowing for backfeed, cf. Chardy et al. (2012) and Hervet and Chardy (2012). Notice that in this case we cannot necessarily guarantee a tree solution with respect to the original network $H$ any more; even worse, if there are cycles, the two-level fiber flow cannot be guaranteed to be integral. The concentrator location and capacity expansion models in Balakrishnan et al. (1991, 1995) and Coyle (1998) all allow for backfeed, but expect a tree network as input. The point-to-multipoint FTTH models in Chardy et al. (2012) and Hervet and Chardy (2012) allow for backfeed (and cycles), but add fiber flow integrality constraints. We remark that when using model (6), (7), (8), (9), (11) and (12), leaving out (10), or when using an undirected formulation in practice, cycles should rarely occur in optimal solutions, since the fixed-charge (trenching) cost $\kappa_{a}$ is typically large compared to the cost for splitters or fiber. 


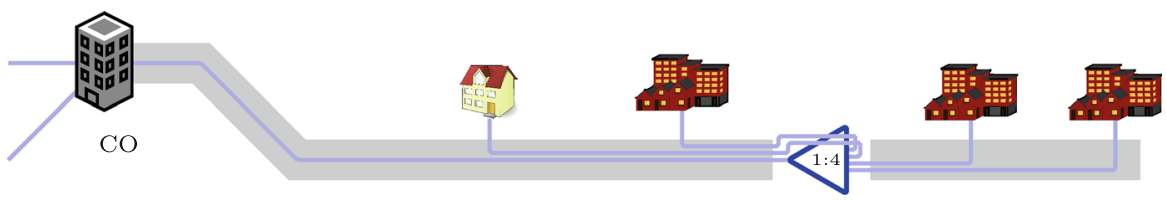

Fig. 4 Customers connected via backfeed: second-level fiber flow connecting the two customer locations on the left runs in the opposite direction to first-level fiber flow on the same arcs in the graph. Compare, in contrast, Fig. 1, where the splitter on the lower right is located between the CO and all of its connected customers. Our models do not support backfeed unless constraints (10) are skipped

Cable and duct installation

In the models above we included a fiber cost term in the objective that assumes a fixed $\operatorname{cost} \bar{\kappa}_{a}$ for providing one fiber on arc $a \in A$ of the network. However, such a cost value is hard to estimate. As mentioned earlier, fibers are not provided individually but come in bundles organized in cables which in turn are aggregated in larger ducts, see Fig. 3. Also the cost for the actual fiber deployment and fiber splicing is ignored in such a simplification. In the following, we introduce a more refined model that is designed to better incorporate the cost for fiber deployment in practice and to provide a more realistic cable and duct installation plan.

We may roughly distinguish two types of cost, first the cost for cables or ducts depending on their length in kilometers, and second the cost for terminating the link equipment, that is, the cost for diverging smaller cables from larger cables, smaller ducts from larger ducts, connecting cables to splitters, OLTs, and ONUs, etc. Here we make the following assumptions which we believe are reasonable in a practical setting:

- We assume a hierarchy of two different types of link equipment to provide fibers (cf. Fig. 3): The fibers themselves are always contained in cables (of different sizes). Cables are always contained in ducts (of different sizes).

- First-level and second-level fiber will never appear in the same cable. That is, we may distinguish first- and second-level cables. However, cables of the different levels may appear in the same duct.

- A subset of the fibers contained in a cable may be diverged by connecting a (potentially smaller) cable and splicing the individual fibers, cf. Fig. 5.

- The cost for connecting smaller cables to larger cables and the cost for connecting cables to COs and splitters is mapped to the (smaller) cable.

- We neglect the cost for connecting the customer to the cable and duct system assuming that this cost is unavoidable.

Notice that (for reasonable cost models) the size of the cables and the size of ducts can only decrease on the path from the $\mathrm{CO}$ to the customer. This is because we only support tree solutions and no backfeed.

With the assumptions above, we also cover the use of micro-cables and microducts, cf. Rigby (2011). Micro-cables, which are essentially fiber bundles, are blown into the micro-duct system and always connect a single customer to the splitter with no interruption. Hence, in our setting, micro-cables can be seen as fibers and micro- 


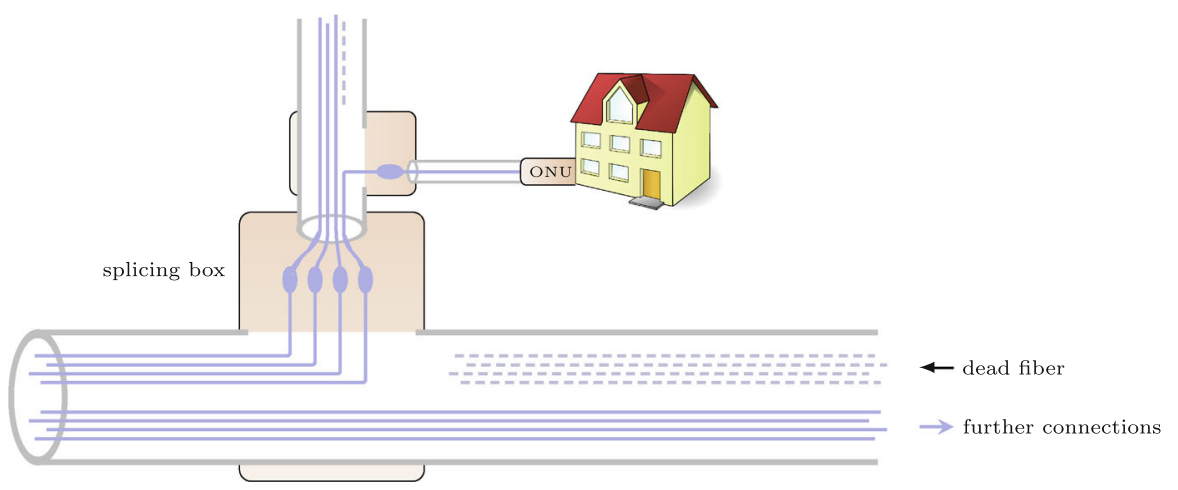

Fig. 5 Smaller cables diverged from larger ones: extracted fibers have to be spliced, their remnants (dead fiber) remain in the cable for the rest of its path, but cannot be used for connections any more

ducts as cables, cf. Fig. 3. In principle, micro-ducts and first-level cables might appear together in the same (second-hierarchy) duct (in contrast to Fig. 3).

Consider the set $\mathcal{P}$ of all paths in the digraph $G=(V, A)$. The set of paths in the subgraph $\left(V \backslash\{r\}, A \backslash A_{0}\right)$ is denoted by $\mathcal{P}^{\prime}$. Given arc $a \in A \backslash A_{0}$, the set of paths in $\mathcal{P}^{\prime}$ containing $a$ is denoted by $\mathcal{P}_{a}^{\prime}$. For every path $p \in \mathcal{P}^{\prime}$, we consider the sets $T_{p}^{1}, T_{p}^{2}$ of installable first-level and second-level cable technologies, respectively. Such a cable technology $t \in T_{p}^{1}$ or $t \in T_{p}^{2}$ might combine multiple cables and it contains $c^{t}>0$ fibers. Installing cable technology $t \in T_{p}^{1}$ or $t \in T_{p}^{2}$ incurs a cost of $\kappa_{p}^{t}>0$ reflecting both, the length-dependent investment cost and the cost for connecting this cable to another (larger) cable, the CO, or a splitter. Similarly, we introduce a set of available duct systems $T_{p}$ for every path $p \in \mathcal{P}^{\prime}$. A duct system $t \in T_{p}$ potentially combines multiple individual ducts and provides capacity for $c^{t}>0$ (first-level or second-level) cables at $\operatorname{cost} \kappa_{p}^{t}>0$, which also refers to investment as well as connection cost. We introduce the following decision variables:

- cable variable $y_{p}^{t} \in\{0,1\}$ deciding whether to install cable technology $t \in T_{p}^{1} \cup T_{p}^{2}$ on path $p \in \mathcal{P}^{\prime}$ or not,

- duct variable $u_{p}^{t} \in\{0,1\}$ deciding whether to install duct technology $t \in T_{p}$ on path $p \in \mathcal{P}^{\prime}$ or not.

A point-to-point or point-to-multipoint FTTH network model with fiber and duct installation plan is then given by:

$$
\min \sum_{a \in A} \kappa_{a} z_{a}+\sum_{i \in V} \kappa_{i} y_{i}+\sum_{p \in \mathcal{P}^{\prime}} \sum_{l=1}^{2} \sum_{t \in T_{p}^{l}} \kappa_{p}^{t} y_{p}^{t}+\sum_{p \in \mathcal{P}^{\prime}} \sum_{t \in T_{p}} \kappa_{p}^{t} u_{p}^{t}
$$

s.t.

$(6)-(12)$

$$
\begin{aligned}
& f_{a}^{l} \leq \sum_{p \in \mathcal{P}_{a}^{\prime}} \sum_{t \in T_{p}^{l}} c^{t} y_{p}^{t} \quad \forall a \in A \backslash A_{0}, l=1,2, \\
& \sum_{p \in \mathcal{P}_{a}^{\prime}} \sum_{l=1}^{2} \sum_{t \in T_{p}^{l}} y_{p}^{t} \leq \sum_{p \in \mathcal{P}_{a}^{\prime}} \sum_{t \in T_{p}} c^{t} u_{p}^{t} \quad \forall a \in A \backslash A_{0},
\end{aligned}
$$




$$
\begin{aligned}
\sum_{t \in T_{p}^{l}} y_{p}^{t} \leq 1 & \forall p \in \mathcal{P}^{\prime}, l=1,2, \\
\sum_{t \in T_{p}} u_{p}^{t} \leq 1 & \forall p \in \mathcal{P}^{\prime}, \\
y_{p}^{t} \in\{0,1\} & \forall p \in \mathcal{P}^{\prime}, t \in T_{p}^{1} \cup T_{p}^{2}, \\
u_{p}^{t} \in\{0,1\} & \forall p \in \mathcal{P}^{\prime}, t \in T_{p} .
\end{aligned}
$$

Constraints (13) ensure that first-level and second-level fiber is covered by first-level and second-level cables, respectively. Constraint (14) ensures that all cables are contained in sufficiently dimensioned duct systems. The generalized upper bound (GUB) constraints (15), (16) and (17) guarantee that only one technology is selected for every potential sub-path.

With such a capacity model, we select one path capacity design out of a finite list of admissble installation scenarios. This is also referred to as a model with explicit capacities, in contrast to a (buy at bulk) model with modular capacities. Capacity models using GUB constraints for telecommunication network design have been introduced by Dahl and Stoer $(1994,1998)$. A discussion of different capacity models can be found, for instance, in Wessäly (2000) and Raack (2012).

In the classical capacitated network design and network loading models (Chopra et al. 1998; Magnanti et al. 1995; Bienstock and Günlük 1996; Raack et al. 2011; Avella et al. 2007), capacities are installed at the network links. Here we install capacity on paths. In this respect, our capacity model resembles approaches to multi-layer telecommunication network design (Orlowski 2009; Idzikowski et al. 2011; Koster et al. 2009) and also models for line planning in public transport networks (Borndörfer et al. 2009; Borndörfer and Karbstein 2012).

Clearly, the above model cannot be applied as it is in practice, due to the exponential number of paths to be considered. A common strategy would be to decompose the planning problem into a first stage which computes a tree topology, and a second stage computing cable and duct installations in the provided tree. Then the model only has to deal with a quadratic number of possible paths, which is much more applicable.

\section{Node installations}

Similar to the fiber equipment installed on the links, we have to provide a more realistic node equipment model and incorporate a more detailed view of the used devices. At the COs outgoing first-level fibers are served, but, clearly, a $\mathrm{CO}$ is not just a single piece of equipment with a maximum fiber capacity coming at a specific cost. The same holds for splitters. Typically there are several capacity expansions possible each of which involves a certain number of devices with certain properties installed in some building. The feasible installations depend on the specific location. We assume a set of available $\mathrm{CO}$ technologies $T_{a}$ associated with each artificial arc $a \in A_{0}$ corresponding to a potential CO location. Technology $t \in T_{a}$ has cost $\kappa^{t}>0$ and a (first-level 
fiber) capacity of $c^{t}$. Installing sufficient capacity at the selected CO locations is then guaranteed by:

$$
\begin{aligned}
\sum_{t \in T_{a}} c^{t} y_{a}^{t} \geq f_{a}^{1} & \forall a \in A_{0}, \\
\sum_{t \in T_{a}} y_{a}^{t} \leq 1 & \forall a \in A_{0}, \\
y_{a}^{t} \in\{0,1\} & \forall a \in A_{0}, t \in T_{a},
\end{aligned}
$$

replacing the capacity constraints (8) for $a \in A_{0}$.

Similar to $\mathrm{CO}$ equipment we can model the splitters at nodes via available technologies. Instead of the integer variable $y_{i}$, a binary variable $y_{i}^{t}$ then decides whether at node $i$ node installation $t \in T_{i}$ is installed at $\operatorname{cost} \kappa_{i}^{t} \geq 0$ or not. Since such a node installation may consist of several splitters (and maybe other devices like joint closures), it comes with a parameter $d_{t}^{1}$ stating how many first-level fibers are demanded at the node in question, if the node installation is chosen. Assuming a fixed splitting ratio of $\theta \in \mathbb{Z}, \theta>0$, the same node installation provides $\theta \cdot d_{t}^{1}$ second-level outgoing fiber. In the above models we have to set

$$
y_{i}=\sum_{t \in T_{i}} d_{t}^{1} y_{i}^{t},
$$

and add the constraints

$$
\begin{array}{ll}
\sum_{t \in T_{i}} y_{i}^{t} \leq 1 & \forall i \in V, \\
y_{i}^{t} \in\{0,1\} & \forall i \in V, t \in T_{i} .
\end{array}
$$

Eventually, the objective has to subsume all costs associated with the different types of technologies, which means that the following terms have to be added:

$$
\sum_{i \in V} \sum_{t \in T_{i}} \kappa^{t} y_{i}^{t}+\sum_{a \in A_{0}} \sum_{t \in T_{a}} \kappa^{t} y_{a}^{t} .
$$

Node technologies have been modeled in a similar way, for instance, by Gualandi et al. (2010). Notice that, in principle, we could also map additional parameters to the technologies, such as power consumption, cable (duct) capacity, volume, the number of holes for cables in joint closures, number of cassette trays, etc. Capacity restrictions can then be modeled more accurately by adding constraints based on these additional parameters. We refrain from detailing this here.

Coverage rates and profits

In practice, it may not be requested to connect $100 \%$ of the households (at once) within a given deployment area to the optical access network. Furthermore, first-level 

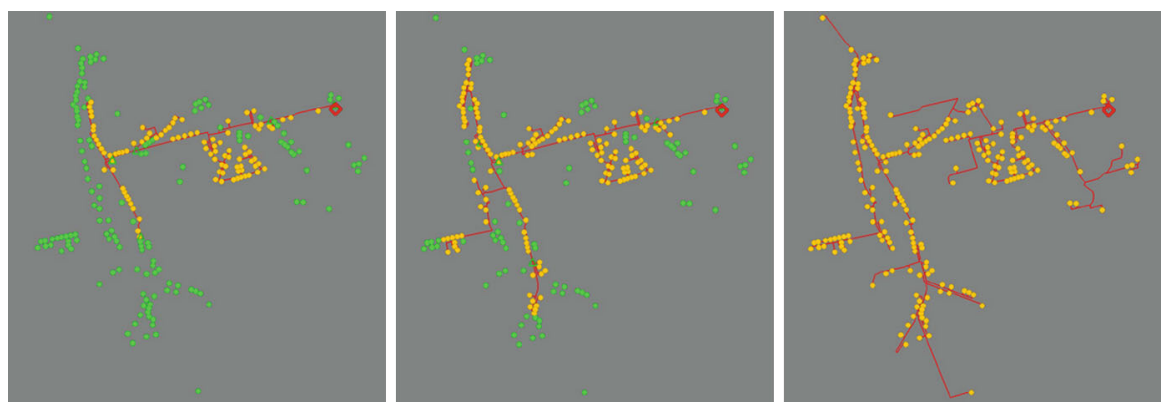

Fig. 6 Networks with coverage rates of 60, 80, and $100 \%$, respectively, for FTTH customers (yellow). (color figure online)

or second-level fiber demand of a customer, building, or neighborhood is typically not a fixed and known number. Instead, network operators might decide about the deployment based on the estimated revenue, trying to balance investment and income within a particular time horizon. In this context, it is also reasonable to study different coverage rates, that is, the percentage of potential customers that will be served by the optical fiber network, cf. Fig. 6. Coverage rates might be based on political regulation, but can also be considered within multi-period planning scenarios, cf. "Deployment over time".

Let us for simplicity come back to the basic model (6), (7), (8), (9), (10), (11) and (12) and let $J \subset V$ be the set of customers with positive demand. We introduce the expected income $\mu_{j}^{1}>0$ and $\mu_{j}^{2}>0$ (over a given time horizon) if customer node $j \in J$ is connected via first-level or second-level fiber, respectively. We also define

- decision variables $x_{i}^{1}, x_{i}^{2} \in\{0,1\}$ for $i \in V$ stating whether or not the node demand at the respective level is satisfied.

Now consider the following extension of model (6), (7), (8), (9), (10), (11) and (12):

$$
\begin{array}{lll}
\min & \sum_{a \in A} \kappa_{a} z_{a}+\sum_{i \in V} \kappa_{i} y_{i}+\sum_{a \in A} \bar{\kappa}_{a}\left(f_{a}^{1}+f_{a}^{2}\right)-\sum_{j \in J} \sum_{l=1}^{2} \mu_{j}^{l} x_{j}^{l} & \\
\text { s.t. } & & \\
\sum_{a \in \delta^{-}(i)} f_{a}^{1}-\sum_{a \in \delta^{+}(i)} f_{a}^{1}=d_{i}^{1} x_{i}^{1}+y_{i} & \forall i \in V \backslash\{r\}, \\
\sum_{a \in \delta^{-}(i)} f_{a}^{2}-\sum_{a \in \delta^{+}(i)} f_{a}^{2} \geq d_{i}^{2} x_{i}^{2}-\theta y_{i} & \forall i \in V \backslash\{r\}, \\
x_{i}^{1}+x_{i}^{2} \leq 1 & \forall i \in V . \\
x_{i}^{1}, x_{i}^{2} \in\{0,1\} & \forall i r .
\end{array}
$$

In the flow constraints (19) and (20) the fiber demand of a customer $i \in V$ is present only if the corresponding decision variable $x_{i}^{1}$ or $x_{i}^{2}$ is switched on. By inequality (21) demand nodes are either connected by first or by second-level signals. The actual 
decision is made by maximizing the estimated revenue. We may use this model together with individual or combined target coverage constraints such as:

$$
\sum_{j \in J} x_{j}^{1} \geq \rho_{1}|J|, \quad \sum_{j \in J} x_{j}^{2} \geq \rho_{2}|J|, \quad \sum_{j \in J}\left(x_{j}^{1}+x_{j}^{2}\right) \geq \rho|J|
$$

with $\rho, \rho_{1}, \rho_{2} \in(0,1]$. This way it can be guaranteed that a predefined percentage of the households or business units in the deployment area is connected with first-level fiber, second-level fiber or either one. Note that in the model above, customers can only be served by either first- or second-level fibers. To relax this, and allow the connection to both levels, as in the original formulation, Constraint (21) can be relaxed or replaced by

$$
x_{i}^{1}=x_{i}^{2}
$$

for certain customer nodes $i$.

Including revenues is done similarly in models for Prize-Collecting Steiner Tree problems and related variants, see, for instance, Ljubić et al. (2006), Costa et al. (2009) and Leitner and Raidl (2011).

\section{FTTC-fiber-to-the-curb}

In case of FTTC approaches, the customers are not directly connected to the optical fiber network, but - using the existing copper cable network - to a DSLAM in a street cabinet, which is then supplied by fiber, see Fig. 1. The street cabinets themselves have to be installed. Since the existing copper infrastructure is predetermined, its topology can be neglected in the optimization model; it merely defines the possible assignments of customers to cabinet nodes, by determining which customers are reachable from a given cabinet within a prescribed distance over the copper network. The decisions to be made are where to open the cabinets and which customer to connect to which cabinet. In combinatorial optimization this is known as a Facility Location problem.

For each customer $j \in J$ we introduce a set $V(j) \subseteq V$ containing all nodes that can be used to open a cabinet (a facility) connecting $j$. Conversely, the set $J(i)$ denotes the set of customers reachable from node $i \in V$ via copper. A customer $j \in J$ now has a demand $b_{j}>0$ in terms of bit rate (instead of fibers, as before), which can be satisfied by connecting him to a cabinet in $V(j)$. An opened cabinet at node $i \in V$ can satisfy the aggregated demand of its connected customers, for which it has a (bit rate) capacity of $c_{i}$. It then creates first-level and second-level fiber demand of $d^{1}$ and $d^{2}$, respectively, depending on the used technology or cabinet type.

Accordingly, the following new variables come into play:

- assignment variables $x_{i j} \in\{0,1\}$ for assigning customers $j \in J$ to their potential cabinets $i \in V(j)$,

- cabinet opening variables $w_{i} \in\{0,1\}$ for nodes $i \in V$. 
Connecting customer $j \in V$ to node $i \in V(j)$ incurs an assignment cost of $\kappa_{i j}>0$. Additionally, street cabinet technology has to be installed at $i$, for which we assume a cost of $\bar{\kappa}_{i}$.

Again we consider an extension of the basic FTTH model (6), (7), (8), (9), (10), (11) and (12):

$$
\begin{array}{cl}
\min & \sum_{a \in A} \kappa_{a} z_{a}+\sum_{i \in V} \kappa_{i} y_{i}+\sum_{a \in A} \bar{\kappa}_{a}\left(f_{a}^{1}+f_{a}^{2}\right)+\sum_{i \in V} \bar{\kappa}_{i} w_{i}+\sum_{j \in J} \sum_{i \in V(j)} \kappa_{i j} x_{i j} \\
\sum_{a \in \delta^{-}(i)} f_{a}^{1}-\sum_{a \in \delta^{+}(i)} f_{a}^{1}=d^{1} w_{i}+y_{i} & \forall i \in V \backslash\{r\}, \\
\sum_{a \in \delta^{-}(i)} f_{a}^{2}-\sum_{a \in \delta^{+}(i)} f_{a}^{2} \geq d^{2} w_{i}-\theta y_{i} & \forall i \in V, \\
\sum_{i \in V(j)} x_{i j}=1 & \forall j \in V, \\
\sum_{j \in J(i)} b_{j} x_{i j} \leq c_{i} w_{i} & \forall i \in V, \\
w_{i}, x_{i j} \in\{0,1\} & \forall j \in V, i \in V(j) .
\end{array}
$$

In contrast to the demand constraints (6) and (7), a fiber demand at node $i \in V$ occurs in constraints (23) and (24) only if a cabinet is opened by setting $w_{i}=1$. That is, compared to the FTTH model, the opened cabinets take the role of fiber customers with node independent fiber demand $d^{1}$ and $d^{2}$. Constraints (25) ensure that every (original) customer is assigned to a cabinet and (26) are the cabinet capacity constraints. This formulation is very general. By adjusting parameters it allows for different use-cases, in particular, for a combination of FTTH and FTTC: If $j \in V(j)$ then customer $j$ is either connected to the optical network via copper infrastructure (FTTC) or directly fed by fiber, if $x_{j j}=1$ (FTTH). We might force the fiber network to reach the customer by setting $V(j):=\{j\}$ and $x_{j j}=1$.

A combination of a facility location and a Steiner tree problem has already been introduced by Gupta et al. (2001) under the term Connected Facility Location. This problem has since been studied often, recently by Gollowitzer and Ljubić (2011) and Arulselvan et al. (2011); Leitner et al. (2013) study a version which allows for mixed FTTH/FTTC deployment. In the literature, the underlying graph is usually extended by introducing assignment edges between customers and their potential cabinets. Assuming a second-level fiber demand of 0 and removing splitters as well second-level flow from the above model, we arrive at the (single-commodity) flow models considered in Gollowitzer and Ljubić (2011) and Ljubić and Gollowitzer (2012). In fact, DSLAMs in a street cabinet in practice are typically directly fed by fiber from the $\mathrm{CO}$, without intermediate splitters (see Rigby 2011), that is, $d^{1}=1$ and $d^{2}=0$.

Similar to the node model for COs and splitter technologies, also the capacity model for street cabinets can be refined. We can assume that the set $T_{i}$ of available technologies at cabinet node $i \in V$ contains possible installations including the necessary 
DSLAM components. Capacity expansion $t \in T_{i}$ has capacity $c^{t}>0$, which can satisfy customer bit rate demand, creates a first- or second-level fiber demand of $d_{t}^{1}$ or $d_{t}^{2}$, respectively, and has cost $\kappa^{t}>0$. Then, by introducing binary variables $y_{i}^{t}$ for the installation of cabinet technology $t \in T$ at node $i \in V$ and by replacing (26) with

$$
\begin{array}{cl}
\sum_{j \in J(i)} b_{j} x_{i j} \leq \sum_{t \in T_{i}} c^{t} y_{i}^{t} & \forall i \in V, \\
\sum_{t \in T_{i}} y_{i}^{t} \leq w_{i} & \forall i \in V,
\end{array}
$$

as well as objective term $\sum_{i \in V} \bar{\kappa}_{i} w_{i}$ with the term $\sum_{i \in V, t \in T_{i}} \kappa^{t} y_{i}^{t}$, the possible cabinet technologies are modeled with much better granularity.

It is also possible to combine the FTTC model with target coverage rates. Adding

$$
\sum_{j \in V} x_{j j} \geq \rho|J|
$$

with $\rho \in(0,1]$ yields a percentage of $\rho \cdot 100 \%$ FTTH customers that are directly connected by optical fiber. As already mentioned, such coverage constraints are commonly used for case studies combined with profits to estimate the revenue of different coverage levels in a particular deployment area, see Fig. 6.

\section{Length restrictions}

An important practical side-constraint results from the fact that the optical signal emitted at the $\mathrm{CO}$ degenerates on its way to the customer, and if it is not regenerated in between, it can travel only a certain maximal distance before it gets undecodable. The technical details about these phenomena are quite involved. The maximal distance that can be achieved depends on the power of the port, the quality of the fiber and the splices, the used bandwidth and bit rate, the splitting ratio, and other factors. Since the allowed length is typically in the range of up to $20 \mathrm{~km}$ (Rigby 2011), for planning in urban areas it can usually be safely ignored, cf. Orlowski et al. (2012). However, in more rural areas it might become critical and should be obeyed.

Even more severe length restrictions come into play if micro-ducts are to be used: micro-cables can only be blown in over a very short distance, typically below $1 \mathrm{~km}$ (Rigby 2011), and if, in addition, concatenating fibers by splicing is forbidden (for cost reasons, for instance) then connecting cables from COs to splitters, as well as from splitters to customers cannot exceed this restrictive value.

One way to deal with maximal connection lengths would be to introduce hopconstraints, restricting the number of links on a path from the $\mathrm{CO}$ to the customer. For Steiner tree problems, such a restriction has been considered for instance by Gouveia (1998, 1999) and Voß (1999). For Connected Facility Location this has been done by Ljubić and Gollowitzer (2012). To use these approaches in our context would require a graph with relatively uniform arc lengths. This could in principle be achieved by 
splitting up long links, blowing up the graph, however. To the best of our knowledge, connectivity problems primarily focusing on length restrictions instead of hop restrictions have not yet been studied.

Length constraints are in general not easy to integrate into arc-flow based models. A way out is to switch to path-based models and consider only suitable subsets of paths. We consider a path decomposition of the Two-level Concentrator Location model (6), (7), (8), (9), (10), (11) and (12) as follows. Given the set $\mathcal{P}$ of all directed paths in $G$, we denote by $\mathcal{P}^{s t} \subseteq \mathcal{P}$ the set of all directed paths from $s$ to $t$ satisfying the length restriction, for $s, t \in V$. We replace the arc flows with a path flow variable $f_{p}$, which counts the number of fibers along path $p \in \mathcal{P}$. If $p \in \mathcal{P}^{r i}$ for $i \in V \backslash\{r\}$, that is, $p$ starts at the root node, then $f_{p}$ corresponds to first-level flow. If, on the other hand, $p \in \mathcal{P}^{i j}$ for $i, j \in V \backslash\{r\}$, then $f_{p}$ corresponds to second-level flow. Then the constraints (10), (11) and (12), augmented with the following, provide a path-based two-level FTTH model.

$$
\begin{aligned}
\sum_{p \in \mathcal{P}^{r i}} f_{p}=d_{i}^{1}+y_{i} & \forall i \in V \backslash\{r\}, \\
\sum_{p \in \mathcal{P}^{i j}} f_{p}=d_{j}^{2} & \forall i, j \in V \backslash\{r\}, \\
\sum_{j \in V \backslash\{r\}, p \in \mathcal{P}^{i j}} f_{p} \leq \theta y_{i} & \forall i \in V \backslash\{r\}, \\
\sum_{p \in \mathcal{P}, a \in p} f_{p} \leq c_{a} y_{a} & \forall a \in A, \\
f_{p} \geq 0 & \forall p \in \mathcal{P} .
\end{aligned}
$$

In this form the model still allows for all possible paths, which will most likely be computationally hazardous (cf. "Cable and duct installation"), if the underlying graph is not sparse-or even a tree. Hence, in practice, a decomposition of the complete problem is advisable in combination with this formulation.

In any case, we may easily restrict the considered path sets $\mathcal{P}^{r i}$ and $\mathcal{P}^{i j}$ to reasonable subsets, which then, however, raises the - rather non-trivial-problem of how to compute these sets. We do not go into further details concerning this question.

\section{Operational constraints}

Depending on the network operator and deployment area, some FTTx networks computed on the basis of the presented models may not necessarily be desirable since they might complicate daily operation and maintenance. For instance, the same customer can be connected to different splitters, which is usually undesired, since it involves higher administrational effort. Many splitter locations with low utilization may be opened if this turns out to be cost-efficient, which, however, leads to higher maintenance costs and a more widespread network. Several publications introduced different (soft) constraints handling different operational aspects, see Balakrishnan et 
al. (1995), Coyle (1998), Flippo et al. (2000), Carpenter et al. (2001) and Hervet and Chardy (2012).

One example is given by the request for contiguity (cf. Balakrishnan et al. 1995): If a customer $j$ is connected to a splitter at node $i$ then all customers on the path from $i$ to $j$ should also be connected to the same splitter node. In our models, this is partly taken care of by the degree constraint (10). These constraints ensure a tree solution and fiber flows in one direction from $\mathrm{CO}$ to the customer. It follows that all customers on the path from the $\mathrm{CO}$ to customer $j$ are at least connected to splitters on that path.

Based on practical requirements, Hervet and Chardy (2012) consider operational constraints relaxing the notion of contiguity: Their splitter delocation rule states that if the highest-level fiber demand of a customer node $j$ exceeds a certain threshold then it must be completely served by a splitter (of a certain level) at the same customer $j$. In our signal-splitting model (with two levels) the same reasoning can be achieved by setting $d_{j}^{2}:=0$ and $d_{j}^{1}:=\tilde{d}_{j}^{1}+\left\lceil\tilde{d}_{j}^{2} / \theta\right\rceil$, if $\tilde{d}_{j}^{l}$ were the original demand values and $\theta$ the splitting ratio. That is, in case there is a large second-level fiber demand at node $j$, we force a connection with enough first-level fibers instead, to which splitters can then be installed directly at $j$.

Similarly, we can integrate the household grouping rule from Hervet and Chardy (2012) stating that if splitters are installed at a certain node, then at least a given minimal number of households should be connected. This essentially avoids underutilization of splitter technologies. In our models we would restrict the available node installations $T_{i}$ at node $i$ to splitter technologies that provide a certain minimal capacity and force a minimal utilization of these technologies. The latter is achieved by explicitly introducing the slack-variable $s_{i} \geq 0$ in constraint (7) by writing

$$
\sum_{a \in \delta^{-}(i)} f_{a}^{2}-\sum_{a \in \delta^{+}(i)} f_{a}^{2}=d_{i}^{2}+s_{i}-\theta y_{i}
$$

with $y_{i}=\sum_{t \in T_{i}} d_{t}^{1} y_{i}^{t}$. Recall that $s_{i}$ corresponds to the number of unused splitter ports at node $i \in V$. If at most $\omega^{t} \geq 0$ ports of technology $t \in T_{i}$ should be unused we may now add

$$
s_{i} \leq \sum_{t \in T_{i}} \omega^{t} y_{i}^{t}
$$

to the model.

On a more general level, operational expenditure (OPEX) can be incorporated into the objective in all models, possibly with inflational correction for a given time horizon. This also accounts for the important issue of energy consumption of the running network. However, estimating the relevant values for the future is subject to high uncertainty (cf. "Data"), which leads into the realms of stochastic optimization. 
Deployment over time

In reality, an access network for a sufficiently large area is usually not built overnight. Budget constraints or shortage of work force might result in the network being developed over months or even years. During this period of time, parts of the network can possibly be used already and generate profit for a carrier, before construction in other sections has even been started. Similarly as for other infrastructure planning problems, models can be time-expanded and turned into incremental models, see, for instance, Chang and Gavish (1995) and Bienstock et al. (2006). As an example, we sketch modifications that can be done to get a multi-period version of the FTTH P2MP model (6), (7), (8), (9), (10), (11) and (12) for $T$ time periods. For an incremental Connected Facility Location problem, relevant for an FTTC scenario, see Arulselvan et al. (2011).

All variables are equipped with a further index $\tau$ to yield variables $f_{a}^{l, \tau}, y_{i}^{\tau}$, and $z_{a}^{\tau}$ representing the temporal state in time step $\tau$, where $\tau \in\{1, \ldots, T\}$. Then the constraints (6), (7), (8), (9), (10), (11) and (12) from the original model, now for every $\tau \in\{1, \ldots, T\}$, state that in each time step the variables determine a concise network. The flow conservation constraints (6), (7) have to be adapted further: By introducing the variable $x_{i}^{\tau} \in\{0,1\}$, we can decide whether node $i$ is connected to the network at time $\tau$, and in (6) and (7) the demands $d_{i}^{1}$ and $d_{i}^{2}$ are replaced by $d_{i}^{1} x_{i}^{\tau}$ and $d_{i}^{2} x_{i}^{\tau}$, respectively, similar as in (19) and (20). Furthermore, the following additional variables are added to the model (cf. Arulselvan et al. 2011):

- $\tilde{f}_{a}^{l, \tau}$ for the number of fibers of level $l$ newly installed on arc $a \in A$ in time step $\tau$,

- $\tilde{y}_{i}^{\tau}$ for $i \in V$, representing the number of splitters newly installed at node $i$ in time step $\tau$,

- $\tilde{z}_{a}^{\tau} \in\{0,1\}$ indicating if in time step $\tau$ the edge corresponding to arc $a$ has to be trenched.

Note that the original variable $z_{a}^{\tau}$ does not indicate trenching, but merely if the arc is "in use" at time $\tau$ or not. This implies that at some point in time before $\tau$ trenching must have happened on $a$; in other words, the following constraint must be satisfied:

$$
z_{a}^{\tau}=\sum_{\sigma=1}^{\tau} \tilde{z}_{a}^{\tau} \quad \forall a \in A, 1 \leq \tau \leq T .
$$

Similar constraints have to be added for the $f$ and $y$ variables. Additionally, we want to make sure that once connected customers are not disconnected again later:

$$
x_{i}^{\tau-1} \leq x_{i}^{\tau} \quad \forall i \in V, 2 \leq \tau \leq T .
$$

Finally, desired coverage rates at intermediate time steps can be specified by

$$
\sum_{i \in V} x_{i}^{\tau} \geq \rho^{\tau}|J|
$$

where $J$ is the set of all customer nodes and $\rho^{\tau}$ is the desired coverage rate in time step $\tau$. A few more intricacies regarding the coupling of $f, \tilde{f}, z$, and $\tilde{z}$ variables have to be 
taken care of, but we do not go into more detail here. The objective then contains-by default — terms for all costs incurred by the installation variables:

$$
\min \sum_{\tau=1}^{T}\left(\sum_{a \in A} \kappa_{a} \tilde{z}_{a}^{\tau}+\sum_{i \in V} \kappa_{i} \tilde{y}_{i}^{\tau}+\sum_{a \in A} \bar{\kappa}_{a}\left(\tilde{f}_{a}^{1, \tau}+\tilde{f}_{a}^{2, \tau}\right)\right)
$$

Additional features that can be taken care of by adapting the objective function are projected future changes in monetary value (by inserting $\tau$-depending weight factors for different costs), maintenance costs for used equipment (by adding terms with $y_{i}^{\tau}$, and possibly $f_{a}^{l, \tau}$ ), and revenue from already connected customers (by subtracting a term with $x_{i}^{\tau}$ ), cf. Arulselvan et al. (2011). In principle, the model can also be extended to account for replacement or removal of equipment, if this seems appropriate, as done in Bienstock et al. (2006).

It is also possible to specify a target network reached at time step $T$ by fixing the variables with time index $\tau=T$ to the appropriate values (cf. Chang and Gavish 1995). Furthermore, if such a terminal state of the network is known beforehand, one can also restrict the underlying network to those nodes and edges that are going to be used in the end. This makes the problem tremendously easier computationally, in particular if the target network is a tree. Note, however, that computing a target network with a $100 \%$ coverage and then restricting the multi-period optimization to the graph determined by the target network might lead to differing solutions if the objective does account for devaluation, maintenance, or customer revenue.

\section{Survivability}

While survivability requirements are essential in the planning of backbone networks, these considerations are a minor issue for fiber optic access networks in practice. Sporadically ring structures are deployed by network operators for feeder cable installations, which would in our models roughly correspond to first-level fibers. In general, models can be adapted to account for two-connectivity in this part of the network. There is also the principal possibility to provide survivable connections for selected customers ("dual homing"), which is implemented by some companies. But, as a matter of fact, all operators implement FTTx access networks as trees (with exceptions in only very special cases), and that is why we do not cover survivability issues here.

\section{Data}

Finally, we briefly address the question concerning what has to be done before optimization can be conducted at all, that is, which kind of information has to be provided to set up an instance of one of the given models and how to obtain it. This step is critical, since, obviously, the more the input data deviates from realistic values, the less reliable optimization solutions will be, even if they are optimal in a mathematical sense. The most decisive pieces of data are described in the following. 


\section{The input graph $H$}

Usually the deployment area in question is given as a geographical region, such as a town, a city district, or a municipality; sometimes its borders are not even clearly specified. To turn this (continuous) shape into a (discrete) graph usable in an algorithmic framework is a highly non-trivial issue. This task may give rise to a multitude of problems from computational geometry or optimization itself. Often geographic information systems (GIS) are used in a process that involves a lot of manual tuning. A convenient way to obtain geographic raw data is to use OpenStreetMap (2013), which may be, however, imprecise and incomplete, due to its open source nature. Google Earth does not provide non-proprietary data, but can be used for visualization, using the $\mathrm{kml}$ format.

Furthermore, once a geographical area is fixed as a graph, further preprocessing may be done, depending on the concrete model to be applied, such as aggregation of customers or edges, or splitting of nodes.

\section{Fiber demand $d_{j}^{l}$ or bit rate demand $b_{j}$}

It is the network operator's decision, with how many fibers or how large bit rate to serve his prospective customers. Still, the precise value for a demand parameter also depends on the size and type of the given location (i.e. the number, and possibly social structure of people using the connection), as well as its importance for the carrier (business units might be more lucrative than households), and potentially other factors. Such information can only be obtained from extensive socio-economic data, which leads to the complicated issue of data privacy and legal regulations which have to be obeyed.

\section{Realistic cost and revenue values}

Often it is not clear how much a certain piece of equipment costs, even more so with the cost of labour for trenching, splicing etc. Standard values can be assumed, which are, however, sometimes not easy to come by. Additionally, bulk purchasing of components and negotiating with suppliers may lead to lower prices. Therefore, a sensitivity analysis of an optimization model with respect to the cost parameters is often desirable to obtain reliable range bounds for the total cost of a network. Even less predictable are values for estimated revenue of (potential) customers.

\section{Existing infrastructure}

If existing infrastructure should be used for the network deployment, information on ducts or dark fiber that is already buried in the ground and can be used-possibly by renting from another company-has to be obtained. However, such information is usually only available to the companies owning the infrastructure, and if so, there is no standard format-and sometimes not much incentive-to provide the data. Hence, similarly to above, a great deal of communication and detailed manual work is indispensable. 


\section{Literature survey}

In the following, we review literature that we believe is relevant in the context of optimizing the deployment of optical access networks. The focus is on algorithmic work and exact approaches. As shown above the resulting optimization problems are combinations of problems in network design and discrete location theory. Of course, we cannot give a full and comprehensive survey about the work that has been done within these areas (and combinations thereof). For general books and collections we refer to Sansó and Sariano (1999), Pióro and Medhi (2004), Resende and Pardalos (2006) and Koster and Muñoz (2009) (network design) and Mirchandani and Francis (1990), Daskin (1995) and Yaman (2005) (location theory); in a recent survey, Contreras and Fernández (2012) give an overview and categorization of various problems ocurring in location and network design. We will instead concentrate on articles with a clear focus on optimization of (optical) access networks and mainly discuss articles that study models similar to those presented in "Modeling FTTx problems". We try to highlight papers that combine network design and discrete location in such a way that the resulting models could, in principle, be a basis for either point-to-point FTTH, point-to-multipoint FTTH, or FTTC planning. Somein particular newer-papers provide computational results, which we gather at the end of the section, see Table 1. Some of these computations are based on real-life data.

\section{Surveys}

The earliest work we mention here is Balakrishnan et al. (1991), a survey on the design of telecommunication access networks, including a wealth of references. Although it contains almost no mathematical formulations, the general concepts and ideas used at the time are presented, such as modeling of traffic concentration and different transmission rates using layered graphs, backfeed, and fixed-charge network design. As such it anticipates concepts like Concentrator Location and Connected Facility Location and gives a comprehensive overview of the types of problems arising in access network planning without explicitly considering fiber optic technology.

Also Gavish (1991) surveys problems that arose in local access network (LAN) design at this time. He presents models and demonstrates algorithms and solution procedures in detail. The considered problems include capacitated spanning tree, multicenter tree network design, and minimum cost loop problems.

The survey article of Gourdin et al. (2002) gathers a great variety of models and applicable solution techniques concerning Facility Location and connection problems, both uncapacitated and capacitated. Also multi-level design problems are covered.

The structural core of the Capacitated Facility Location Problem, as it appears as part of our models in "FTTC-fiber-to-the-curb", is discussed in Cornuéjols et al. (1991) and Sridharan (1995). Additionally valid inequalities, different relaxations, decomposition methods, and complexity results are given there. 
Single-level network design

As shown in "Modeling FTTx problems", point-to-point FTTH deployment (ignoring splitting) is a single-level, single-source, multiple-target network design problem. In the uncapacitated case, multiple clients are connected to a central office or a root node representing different COs using a Steiner tree. In the capacitated case, sufficient edge (and possibly node) capacity is provided in addition to satisfy the clients' fiber demand. For the pure Steiner tree problem in graphs, we refer the interested reader to Hwang and Richards (1992), Chopra and Rao (1994a,b) and Koch and Martin (1998). In Orlowski et al. (2012) the authors estimate the trenching cost for FTTx deployment using a Steiner tree model.

Cruz et al. (1998) study a single-source uncapacitated network design formulation that is close to formulation (1), (2), (3), (4) and (5). They develop a branch-and-bound algorithm based on a Lagrangian relaxation. Motivated by local access networks, Randazzo and Luna (2001) compare single-commodity and multi-commodity formulations for the same problem and use Benders decomposition as well as Lagrangian relaxation to solve it. They incorporate fixed costs for opened arcs and variable costs for the amount of (fiber) flow on arcs. Also Ortega and Wolsey (2003) study a similar single-commodity uncapacitated fixed-charge network design problem. They provide strong valid inequalities and a branch-and-cut algorithm.

The capacitated case with edge (cable) capacities is also known as network loading or (if cable capacities obey economies of scale) buy-at-bulk network design. In the case of a single source node, the problem is often referred to as the LAN design problem. Chopra et al. (1998) prove NP-hardness of single-source single-sink network design. Salman et al. (2008), and similarly Raghavan and Stanojević (2006), provide a special branch-and-bound algorithm for LAN design based on approximating the capacity step cost function by its lower convex envelope. Similar to Randazzo and Luna (2001), Ljubić et al. (2012) compare different formulations and solution methods for LAN design based on disaggregating the commodities and using Benders decomposition.

Notice that we ignore the wealth of literature about the more general multicommodity (multiple-sources, multiple-sinks) capacitated network design problem here, which does not reflect the special characteristics of P2P FTTH design. For general capacitated network design, we refer to the books mentioned above and to Raack (2012) for a comprehensive introduction.

\section{Multi-level network design}

Recall that one of the essential aspects in point-to-multipoint FTTH planning as in model (6), (7), (8), (9), (10), (11) and (12) is the notion of splitters and splitting ratios. Splitters act as signal concentrators and can be seen as transition nodes between two different levels of the overall (tree) network. The first level connects all splitters to the central office. The second level connects all customers to splitters. In this respect, P2MP FTTH planning can be seen as a capacitated multi-level network design problem. 
The study of multi-level network design has been initiated by Current et al. (1986), Current and Pirkul (1991), and Balakrishnan et al. (1994a,b), motivated by problems in the topological design of hierarchical communication and transportation networks. Depending on whether there is to decide only about the assignment of nodes to other nodes or devices, or there is an underlying meshed graph, in which topology decisions have to be made, the problems differ considerably, both with respect to models, as well as computational tractability. There is a great variety of research in this direction considering different aspects and different topologies such as star-star, tree-star, treetree, or ring-star topologies, see for instance Chopra and Tsai (2002), Labbé et al. (2004), Labbé and Yaman (2008), Gollowitzer et al. (2013b) and the references therein. These papers, however, mainly concentrate on topological decisions and ignore the notion of client demand and signal concentration at the transition nodes.

Balakrishnan et al. (1991, 1995) introduce a LAN Expansion Planning Problem: given a rooted tree containing the $\mathrm{CO}$ and all customers, together with projected demands, install enough capacity on edges and in nodes to route the demands, minimizing total cost. At nodes, concentrators can be installed which act similarly to splitters in FTTx networks. In particular, the authors introduce the trade-off between signal concentration and cable installation. They also consider contiguity constraints and discuss backfeed. Models are presented and solution approaches are discussed. In fact, Balakrishnan et al. (1991) provide the basis for the multi-level flow formulation with splitting ratios we use in "Modeling FTTx problems".

Also Flippo et al. (2000) study a concentrator problem with two flow levels, where concentrating devices are installed in some nodes of a given tree, which then serve the customers. Here also contiguity, as well as non-bifurcated routing (meaning that the complete demand for a customer may only be served by one concentrator) is required.

In Balakrishnan et al. (1991, 1995) and Flippo et al. (2000) the given network already has tree structure, which simplifies the problem and gives rise to dynamic programming approaches.

In contrast, Randazzo et al. (2001) consider a general underlying network and study a two-level problem motivated by the mixed planning of optical and copper networks. They use a flow formulation similar to the one in "Modeling FTTx problems", but without signal concentration and splitting ratios. Capacity at the transition nodes is ignored. The authors present a solution approach based on Benders decomposition.

Also Cruz et al. (2003) model a network design problem with multiple levels using a multi-level flow formulation. In the form described, the problem applies to the planning of networks with multiple connection types incurring different variable costs on arcs, such as fiber and copper connections. Accordingly the authors provide a case study of a Brazilian town, where a fiber/copper network had to be deployed by a telecommunications company and observe that, if costs for optical technology are reasonably low in comparison to those for copper-based one-which is generally the case today-, then there is no incentive to deploy copper any more.

Kim et al. (2011) present models that allow for FTTH P2MP planning with two splitting levels. The models incorporate the placement of splitters at nodes, and additionally the selection of cables on edges of the given underlying tree, without explicitly accounting for splicing costs, however. Some valid inequalities are derived, linearizations of the step function for cable costs, and heuristics are given. 
Even closer to our formulation for FTTH with splitting is the model presented by Chardy et al. (2012). They use an undirected flow formulation similar to ours in "Modeling FTTx problems" and to the one in Balakrishnan et al. (1991), allow two splitter levels, that is, three levels of flow, and explicitly count free splitter ports at nodes. Additionally, they provide valid inequalities for the model and discuss graph reductions that prove effective for applying the IP model to practical instances.

The same model, generalized to an arbitrary number of splitter levels, is used in Hervet and Chardy (2012). In this work, special focus is on operational constraints, see "Operational constraints". Various rules, aimed at decreasing operations and management efforts during runtime of the network, are formulated and integrated into the formulation as an IP.

The two-level model considered by Bley et al. (2013) essentially coincides with the one we are using in "Signal splitting". In addition, arc usage by first- and second-level fibers is distinguished and capacities for splitter nodes and COs are included. For this model the authors provide two alternative Langrangian decomposition approaches: one decomposing into first and second level, the other into fixed-charge and flow-cost part. Furthermore, valid inequalities and heuristics are given.

\section{Network design and facility location}

Whenever equipment, such as splitters, has to be installed at the transition nodes in multi-level network design, as in P2MP FTTH, the problem contains the well-known Facility Location problem as a special case. Even closer to classical Facility Location is the problem of opening DSLAMs in street cabinets and assigning customers to these facilities in FTTC deployment planning.

Sherali et al. (2000) study the access network design problem as an assignment problem on a bipartite graph, similarly to Cornuéjols et al. (1991), but considering the possibility that demand can be satisfied directly at the $\mathrm{CO}$, as well as special additional costs. They provide reformulations and primal heuristics for this model.

Carpenter et al. (2001) is among the earliest papers to explicitly mention FTTx network planning. They state IP models for the assignment of customers to facility nodes that incorporate capacities of different DSLAM node installations. Similarly as in "FTTC-fiber-to-the-curb", customers within a certain range limit of each facility can be connected, and one node installation out of a list of available ones has to be chosen in a GUB constraint, as in "Node installations". A number of issues is ignored, most notably assignment costs and connectivity between the facilities- the underlying network is assumed to be a tree. However, some contiguity issues are treated, and possible extensions, such as more sophisticated node installations and profit maximization are mentioned. The models are solved with a dynamic programing approach, and not by solving the IPs.

Gualandi et al. (2010) model the FTTH problem with one splitting level as an assignment problem using a tripartite graph. Their model can be adapted to account for an FTTC scenario and also includes the use of node technologies. Instead of solving the IPs, they propose LP-based and Constraint Programming approaches, for which they also present results of computations. 
In Eira et al. (2012) a Facility Location model for the assignment of ONUs to splitters with different splitting ratios is presented, where splitting can be realized freely over multiple levels. The actual network topology is ignored in this model, which means that trenching and cable cost cannot be properly included. However, the authors try to incorporate operational expenses over the expected running time of the network into the assignment costs. Besides a computational comparison between an IP model and a heuristic, the authors also report on the sensitivity of the results with respect to these uncertain cost values.

Gupta et al. (2001) introduced the term Connected Facility Location for a particular generalization of the Facility Location Problem. In addition to assigning customers to facilities, the facilities have to be interconnected by a Steiner tree. In this respect, Connected Facility Location is a two-level network design problem requiring a tree-star topology. However, in its capacitated form the problem models the FTTC deployment, where the Steiner tree in the first level corresponds to the fiber connection (without splitting) between the COs and the DSLAMs (the facilities) and the second level corresponds to the assignment of customers to the DSLAMs.

The probably most comprehensive study of Connected Facility Location models was done by Gollowitzer and Ljubić (2011). They give a reformulation for directed graphs and present many different formulations: cut-based models, flow formulations using single- and multi-commodity flow, to customers, as well as to facilities, models using common flow variables, sub-tour elimination constraints, and Miller-Tucker-Zemlin constraints. All formulations are compared with respect to their polyhedral strength and separation and heuristic procedures for branch-and-cut are discussed.

In Ljubić and Gollowitzer (2012) various ways to model hop-constraints for the Connected Facility Location problem are examined. All formulations use layered graphs of some kind, together with preprocessing steps to reduce the size of the graph. Again a polyhedral comparison of the models is given and various methods for separating cuts are presented.

This work is extended in Gollowitzer et al. (2013a) to Capacitated Connected Facility Location. An IP formulation is presented, which is essentially our FTTC model (8), (9), (10), (11), (12), (23), (24), (25), (26) and (27) without second-level fiber flow and optical splitters. The authors develop strong valid inequalities and a branch-and-cut algorithm, as well as computations based on real-life FTTC and FTTB instances, see below.

Models for Capacitated Connected Facility Location that take profits generated by customers into account are studied in Leitner and Raidl (2011). The paper exhibits a branch-and-cut algorithm with additional column generation of assignment pattern variables. Additionally, cut-based and flow models are compared, both theoretically, with respect to the strength of the LP relaxation, and computationally.

Finally, a problem which realizes a slightly different variant of FTTC, but still has some similarities to Connected Facility Location is considered by Gupta and Pirkul (2000) and Patterson and Rolland (2002). They aim at creating a network with optical cables feeding ONUs from the central office and co-axial TV cables connecting the customers with the ONUs. However, in this case the first level optical fiber topology is 
assumed to be a star, while customers are connected by co-axial subtrees to the optical network, that is, a star-tree topology is constructed.

\section{Computational studies}

Table 1 gives a summary and rough comparison of computations that explicitly refer to FTTx planning. Surely, individual results are hard to compare, due to different hardware used, different intentions for which the computations were made, and, of course, differences in the models considered. Hence, we do not give any precise numbers, but rather sketch the sizes of instances that can be treated (to whatever end) with the presented approaches.

The columns describing the various features try to categorize the referred work; note that this does not mean that the models presented there can be directly applied to the given problem, nor are they necessarily comparable to the models given in "Modeling FTTx problems".

Whenever models explicitly involve a graph, the sizes of these within the treated instances are given in columns "\# Nodes" and "\# Edges". Whenever there is a facility location subproblem involved, the numbers of potential facility nodes is given in "\# facilities". Similarly, the column "\# customers" indicates the numbers of customers. Depending on the model, customers may be a subset of all nodes or not: whenever customers are only connected to the graph via assignment edges (cf. "FTTC-fiberto-the-curb"), they do not count towards the number of graph nodes stated in column "\# Nodes".

In the columns describing the instance types, "Real-life" does not necessarily mean that the instances, as they were used for the computations, are also used by network operators; this is rarely the case, since usually companies are quite reluctant to give away their exact planning data. Furthermore, the format in which such data is available - if at all-is not directly applicable to an optimization model, so that it has to be adapted anyway. In contrast, "non-random" instances mean those that are not derived from real cases, but try to mimic decisive properties, e.g. by using GIS information for a (randomly) selected geographical area.

\section{Summary}

FTTx network planning is the task to solve all problems arising in the context of connecting all (or some) customers in a (relatively small) deployment area to one or more central offices using optical fiber (and potentially existing copper cables) and possibly passive concentrators in such a way that many side-constraints are satisfied and certain costs are minimized. This topic is currently a mathematically hot issue since the roll-out of such networks has begun the world over, and network providers are seeking for means to keep the very high costs for establishing such an infrastructure under control.

Using inspirations from a large number of papers that have been written on special aspects of the problem and surveying their contents we have, starting from a basic model, incrementally designed a mathematical model of FTTx network planning that 


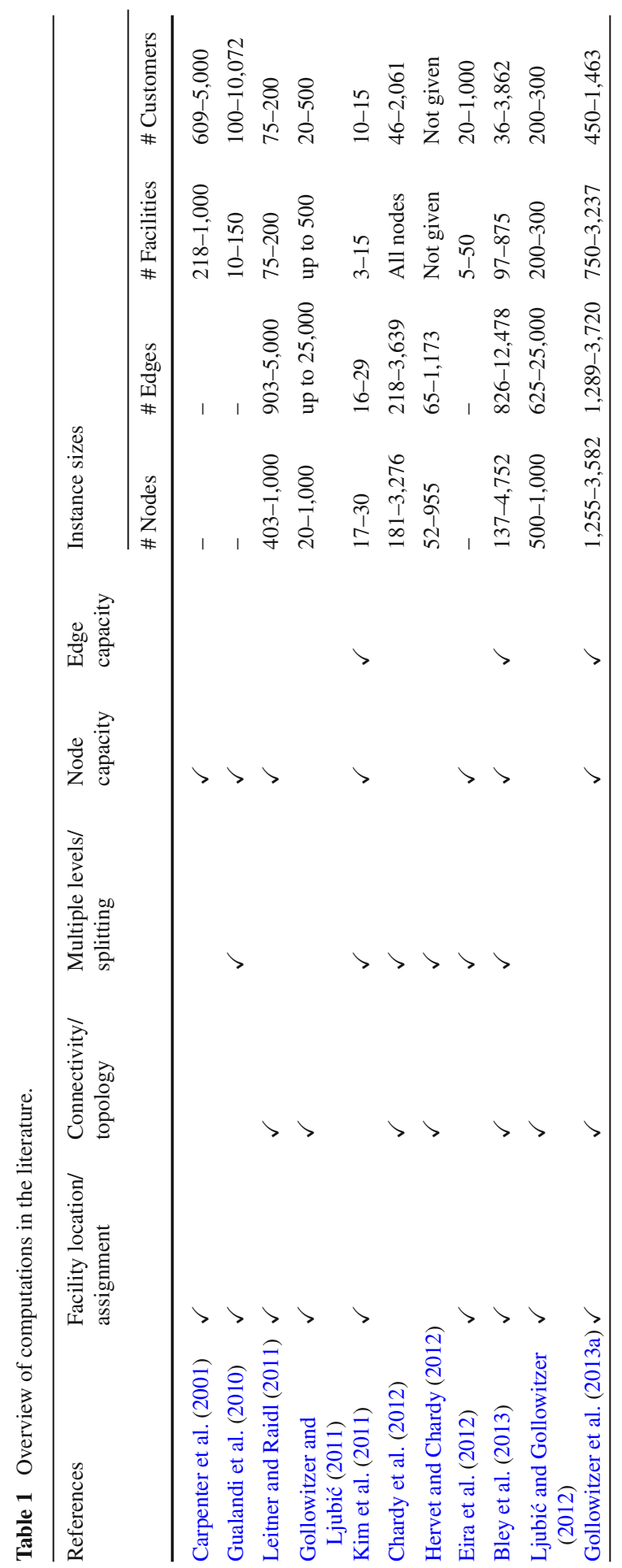




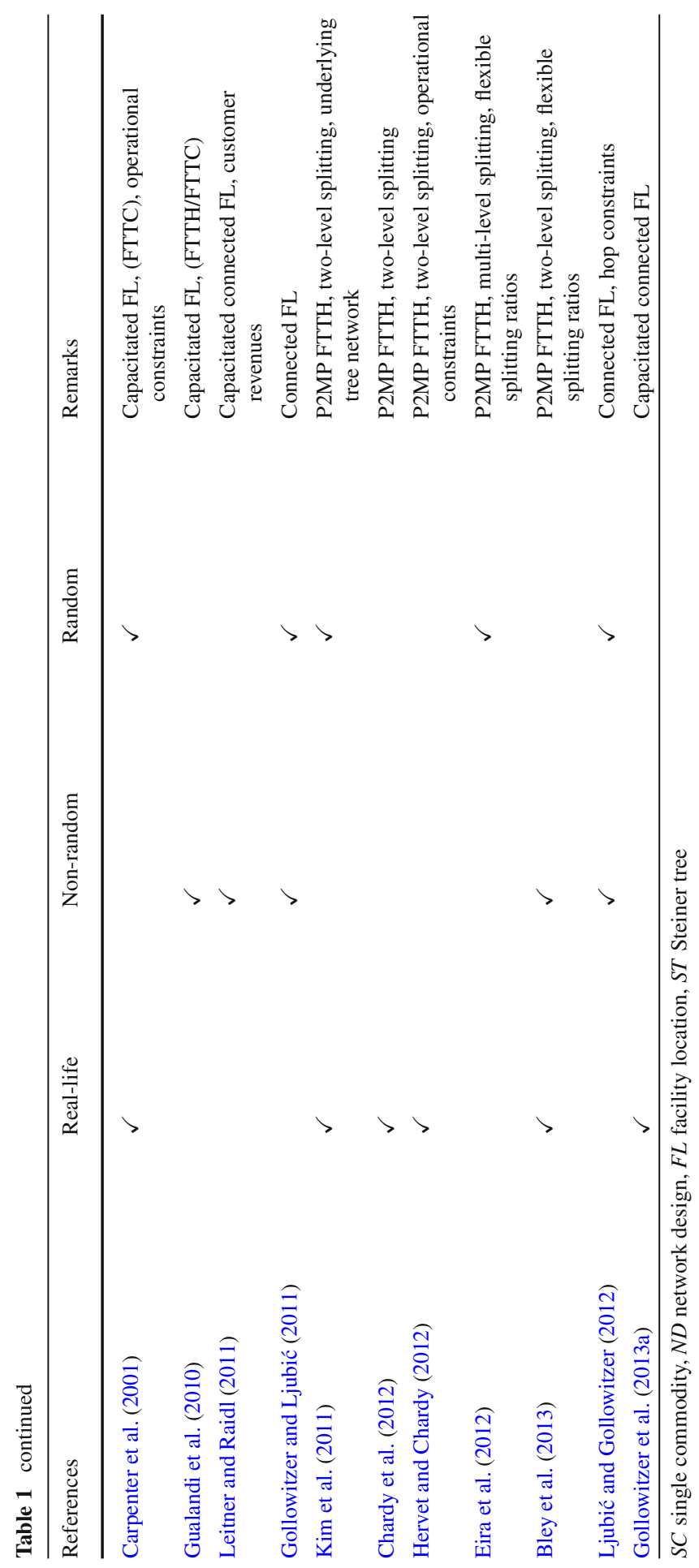


takes almost all aspects of this complex task into account. In its full scale, this model is not solvable for realistic cases. However, we indicate (and mention associated computational studies) how simplifications of this general model can be employed to support the associated complex decision making. We are convinced that proper implementations of our model will be of significant help in FTTx network planning.

Acknowledgments This research has been supported by the DFG, within the Research Center Matheon and the D-A-CH framework, and by BMBF, within the "KMU-innovativ" framework. For fruitful discussions and comments improving this work, the authors thank Roland Wessäly, Sebastian Orlowski, and Andreas Bley, as well as the reviewers for corrections and valuable suggestions for improvements.

\section{References}

Arulselvan A, Bley A, Gollowitzer S, Ljubić I, Maurer O ( 2011) MIP modeling of incremental connected facility location. In: Proceedings of the 5th international network optimization conference (INOC). Lecture notes in computer science, vol. 6701, pp 490-502

Avella P, Mattia S, Sassano A (2007) Metric inequalities and the network loading problem. Discret Optim 40(1):103-114

Balakrishnan A, Magnanti TL, Shulman A, Wong RT (1991) Models for planning capacity expansion in local access telecommunication networks. Ann Oper Res 33:239-284

Balakrishnan A, Magnanti TL, Mirchandani P (1994a) A dual-based algorithm for multi-level network design. Manag Sci 400(5):567-581

Balakrishnan A, Magnanti TL, Mirchandani P (1994b) Modeling and heuristic worst-case performance analysis of the two-level network design problem. Manag Sci 40:846-867

Balakrishnan A, Magnanti TL, Wong RT (1995) A decomposition algorithm for local access telecommunications network expansion planning. Oper Res 430(1):58-76

Bienstock D, Günlük O (1996) Capacitated network design-polyhedral structure and computation. INFORMS J Comput 8:243-259

Bienstock D, Raskina O, Saniee I, Wang Q (2006) Combined network design and multiperiod pricing: modeling, solution techniques, and computation. Oper Res 540(2):261-276

Bley A, Ljubić I, Maurer O (2013) Lagrangian decompositions for the two-level FTTx network design problem. Eur J Comput Optim doi:10.1007/s13675-013-0014-z

Borndörfer R, Karbstein M (2012) A direct connection approach to integrated line planning and passenger routing. In: Delling D, Liberti L (eds) Proceedings of the 12th workshop on algorithmic approaches for transportation modelling, optimization, and systems (ATMOS 2012). Open access series in informatics, vol. 25, pp 47-57

Borndörfer R, Neumann M, Pfetsch M (2009) The line connectivity problem. In: Fleischmann B, Borgwardt KH, Klein R, Tuma A (eds) Operations research proceedings 2008, pp 557-562, Springer Verlag, New York

Carpenter T, Eiger M, Shallcross D, Seymour P (2001) Node placement and sizing for copper broadband access networks. Ann Oper Res 106:199-228

Chang S-G, Gavish B (1995) Lower bounding procedures for multiperiod telecommunications network expansion problems. Oper Res 430(1):43-57

Chardy M, Costa M-C, Faye A, Trampont M (2012) Optimizing splitter and fiber location in a multilevel optical FTTH network. Eur J Oper Res 2220(3):430-440

Chopra S, Rao MR (1994a) The Steiner tree problem I: formulations, compositions and extension of facets. Math Program 640(1):209-229

Chopra S, Rao MR (1994b) The Steiner tree problem II: properties and classes of facets. Math Program 640(1):231-246

Chopra S, Tsai C-Y (2002) A branch-and-cut approach for minimum cost multi-level network design. Discret Math 2420(1-3):65-92

Chopra S, Gilboa I, Sastry ST (1998) Source sink flows with capacity installation in batches. Discret Appl Math 86:165-192

Contreras I, Fernández E (2012) General network design: a unified view of combined location and network design problems. Eur J Oper Res 219:680-697 
Cornuéjols G, Sridharan R, Thizy J-M (1991) A comparison of heuristics and relaxations for the capacitated plant location problem. Eur J Oper Res 50:280-297

Costa AM, Cordeau J-F, Laporte G (2009) Models and branch-and-cut algorithms for the Steiner tree problem with revenues, budget and hop constraints. Networks 530(1):141-159

Coyle A (1998) An algorithm for capacity expansion in local access networks. In: Proceedings of the 33rd annual conference of the Operations Research Society of New Zealand. http://www.orsnz.org.nz/conf33/ papers/p99.pdf

Cruz F, Mateus G, MacGregor Smith J (1998) Solving to optimality the uncapacitated fixed-charge network flow problem. Comput Oper Res 25:67-81

Cruz F, Mateus G, MacGregor Smith J (2003) A branch-and-bound algorithm to solve a multi-level network optimization problem. J Math Model Algorithms 2:37-56

Current JR, Pirkul H (1991) The hierarchical network design problem with transshipment facilities. Eur J Oper Res 520(3):338-347

Current JR, ReVelle CS, Cohon JL (1986) The hierarchical network design problem. Eur J Oper Res 270(1):57-66

Dahl G, Stoer M (1994) A polyhedral approach to multicommodity survivable network design. Numerische Mathematik 68:149-167

Dahl G, Stoer M (1998) A cutting plane algorithm for multicommodity survivable network design problems. INFORMS J Comput 10:1-11

Daskin MS (1995) Network and discrete location: models, algorithms, and applications. John Wiley, London

Eira A, Pedro J, Pires J (2012) Optimized design of multistage passive optical networks. J Opt Commun Netw 40(5):402-411

Flippo OE, Kolen AW, Koster AM, van de Leensel FL (2000) A dynamic programming algorithm for the local access telecommunication network expansion problem. Eur J Oper Res 127:189-202

Garey MR, Johnson DS (1979) Computers and intractability: a guide to the theory of NP-completeness. Freeman and Company, New York

Gavish B (1991) Topological design of telecommunication networks-local access design methods. Ann Oper Res 33:17-71

Gollowitzer S, Ljubić I (2011) MIP models for connected facility location: a theoretical and computational study. Comput Oper Res 38:435-449

Gollowitzer S, Gendron B, Ljubić I (2013a) A cutting plane algorithm for the capacitated connected facility location problem. Comput Optim Appl doi:10.1007/s10589-013-9544-9

Gollowitzer S, Gouveia L, Ljubić I (2013b) Enhanced formulations and branch-and-cut for the two level network design problem with transition facilities. Eur J Oper Res 2250(2):211-222

Gourdin E, Labbé M, Yaman H (2002) Telecommunication and location. In: Drezner Z, Hamacher H (eds) Facility location: applications and theory, chapt. 9, pp 275-305, Springer, New York

Gouveia L (1998) Using variable redefinition for computing lower bounds for minimum spanning and Steiner trees with hop constraints. INFORMS J Comput 10:180-188

Gouveia L (1999) Using hop-indexed models for constrained spanning and Steiner tree models. In: Sansò B, Soriano P (eds) Telecommunications network planning, chapt. 2, pp 21-32. Kluwer Academic Publishers, London

Gualandi S, Malucelli F, Sozzi DL (2010) On the design of the next generation access networks.In: Proceedings of the CPAIOR 2010. Lecture notes in computer science, vol 6140, pp162-175

Gupta A, Kleinberg J, Kumar A, Rastogi R, Yener B (2001) Provisioning a virtual private network: a network design problem for multicommodity flow. In: Proceedings of the 33 annual ACM symposium on theory of computing (STOC 2001), pp 389-398

Gupta R, Pirkul H (2000) Hybrid fiber co-axial CATV network design with variable capacity optical network units. Eur J Oper Res 123:73-85

Hervet C, Chardy M (2012) Passive optical network design under operations administration and maintenance considerations. J Appl Oper Res 40(3):152-172

Hwang FK, Richards DS (1992) Steiner tree problems. Networks 220(1):55-89

Idzikowski F, Orlowski S, Raack C, Woesner H, Wolisz A (2011) Dynamic routing at different layers in IP-over-WDM networks-maximizing energy savings. Opt Switch Netw Special Issue Green Commun 80(3):181-200

Keiser G (2006) FTTX concepts and applications. Wiley series in telecommunications and signal processing. John Wiley, London 
Kim Y, Lee Y, Han J (2011) A splitter location-allocation problem in designing fiber optic access networks. Eur J Oper Res 2100(2):425-435

Koch T, Martin A (1998) Solving Steiner tree problems in graphs to optimality. Networks 32:207-232

Koster AMCA, Muñoz X (2009) Graphs and algorithms in communication networks. Springer, New York

Koster AMCA, Orlowski S, Raack C, Baier G, Engel T, Belotti P (2009) Branch-and-cut techniques for solving realistic two-layer network design problems. In: Koster AMCA, Muñoz X (eds) Graphs and algorithms in communication networks, 3rd edn. Springer, New York, pp 95-118

Labbé M, Yaman H (2008) Solving the hub location problem in a star-star network. Networks 510(1):19-33

Labbé M, Laporte G, Martín IR, González JJS (2004) The ring star problem: polyhedral analysis and exact algorithm. Networks 430(3):177-189

Leitner M, Raidl GR (2011) Branch-and-cut-and-price for capacitated connected facility location. J Math Model Algorithms 10:245-267

Leitner M, Ljubić I, Sinnl M, Werner A (2013) On the two-architecture connected facility location problem. Electron Notes Discret Math 41:359-366

Ljubić I, Gollowitzer S (2013) Layered graph approaches to the hop constrained connected facility location problem. INFORMS J Comput 250(2):256-270 doi:10.1287/ijoc.1120.0500

Ljubić I, Weiskircher R, Pferschy U, Klau GW, Mutzel P, Fischetti M (2006) An algorithmic framework for the exact solution of the prize-collecting Steiner tree problem. Math Program 105:427-449

Ljubić I, Putz P, Salazar-González J-J (2012) Exact approaches to the single-source network loading problem. Networks 59:89-106

Magnanti TL, Mirchandani P, Vachani R (1995) Modelling and solving the two-facility capacitated network loading problem. Oper Res 43:142-157

Mirchandani PB, Francis RL (1990) Discrete location theory. John Wiley, New York

OpenStreetMap Project (2013) http://www.openstreetmap.org. Accessed 20 Nov 2013

Orlowski S (2009) Optimal design of survivable multi-layer telecommunication networks. PhD thesis, Technische Universität Berlin, Berlin

Orlowski S, Pióro M, Tomaszewski A, Wessäly R (2010) SNDlib 1.0—survivable network design library. Networks 550(3):276-286. http://sndlib.zib.de/. Accessed 20 Nov 2013

Orlowski S, Werner A, Wessäly R (2011) Estimating trenching costs in FTTx network planning. In: Klatte D, Lüthi H-J, Schmedders K (eds) Operations research proceedings 2011, pp 89-94, Springer Verlag, New York

Ortega F, Wolsey LA (2003) A branch-and-cut algorithm for the single-commodity, uncapacitated, fixedcharge network flow problem. Networks 41:143-158

Patterson RA, Rolland E (2002) Hybrid fiber coaxial network design. Oper Res 500(3):538-551

Pióro M, Medhi D (2004) Routing, flow, and capacity design in communication and computer networks. Morgan Kaufmann Publishers, Burlington

Raack C (2012) Capacitated network design-multi-commodity flow formulations, cutting planes, and demand uncertainty. PhD thesis, Technische Universität Berlin, Berlin

Raack C, Koster AMCA, Orlowski S, Wessäly R (2011) On cut-based inequalities for capacitated network design polyhedra. Networks 570(2):141-156

Raghavan S, Stanojević D (2006) A note on search by objective relaxation. In: Raghavan S, Anandalingam $\mathrm{G}$ (eds) Telecommunications planning: innovations in pricing, network design and management, vol. 33 of Operations research/computer science interfaces, pp 181-201, Springer, New York

Randazzo C, Luna H (2001) A comparison of optimal methods for local access uncapacitated network design. Ann Oper Res 106:263-286

Randazzo C, Luna H, Mahey P (2001) Benders decomposition for local access network design with two technologies. Discret Math Theor Comput Sci 4:235-246

Resende MGC, Pardalos PM (2006) Handbook of optimization in telecommunications. Springer, New York

Rigby P (ed) (2011) FTTH handbook 4th edn. FTTH Council Europe,

Salman FS, Ravi R, Hooker JN (2008) Solving the capacitated local access network design problem. INFORMS J Comput 20:243-254

Sansó B, Sariano P (1999) Telecommunications network planning. Kluwer Academic Publishers, London

Sherali HD, Lee Y, Park T (2000) New modeling approaches for the design of local access transport area networks. Eur J Oper Res 127:94-108

Sridharan R (1995) The capacitated plant location problem. Eur J Oper Res 87:203-213

Voß S (1999) The Steiner tree problem with hop constraints. Ann Oper Res 86:321-345 
Wessäly R (2000) Dimensioning survivable capacitated networks. PhD thesis, Technische Universität Berlin, Berlin

Yaman H (2005) Concentrator location in telecommunication networks. Combinatorial optimization. Springer, New York 